\title{
Temporally Altered miRNA Expression in a Piglet Model of Hypoxic Ischemic Brain Injury
}

\author{
Sophie Casey ${ }^{1,2,3}$ (1D $\cdot$ Kate Goasdoue ${ }^{4} \cdot$ Stephanie M. Miller ${ }^{4} \cdot$ Gary P. Brennan ${ }^{5} \cdot$ Gary Cowin $^{6} \cdot$ Adam G. O'Mahony $^{3}$. \\ Christopher Burke $^{7}$ - Boubou Hallberg ${ }^{8}$. Geraldine B. Boylan ${ }^{1}$. Aideen M. Sullivan ${ }^{3}$ - David C. Henshall ${ }^{5,9}$. \\ Gerard W. O'Keeffe $^{1,3} \cdot$ Catherine Mooney ${ }^{1,9,10} \cdot$ Tracey Bjorkman $^{4} \cdot$ Deirdre M. Murray ${ }^{1,2}$
}

Received: 15 April 2020 / Accepted: 8 July 2020 / Published online: 27 July 2020

(C) Springer Science+Business Media, LLC, part of Springer Nature 2020

\begin{abstract}
Hypoxic ischemic encephalopathy (HIE) is the most frequent cause of acquired infant brain injury. Early, clinically relevant biomarkers are required to allow timely application of therapeutic interventions. We previously reported early alterations in several microRNAs (miRNA) in umbilical cord blood at birth in infants with HIE. However, the exact timing of these alterations is unknown. Here, we report serial changes in six circulating, cross-species/bridging biomarkers in a clinically relevant porcine model of neonatal HIE with functional analysis. Six miRNAs - miR-374a, miR-181b, miR-181 a, miR-151a, miR-148a and miR128 - were significantly and rapidly upregulated 1 -h post-HI. Changes in miR-374a, miR-181b and miR-181a appeared specific to moderate-severe HI. Histopathological injury and five miRNAs displayed positive correlations and were predictive of MRS $\mathrm{Lac} / \mathrm{Cr}$ ratios. Bioinformatic analysis identified that components of the bone morphogenic protein (BMP) family may be targets of miR-181a. Inhibition of miR-181a increased neurite length in both SH-SY5Y cells at 1 DIV (days in vitro) and in primary cultures of rat neuronal midbrain at 3 DIV. In agreement, inhibition of miR-181a increased expression of BMPR2 in differentiating SH-SY5Y cells. These miRNAs may therefore act as early biomarkers of HIE, thereby allowing for rapid diagnosis and timely therapeutic intervention and may regulate expression of signalling pathways vital to neuronal survival.
\end{abstract}

Keywords Hypoxic ischemic encephalopathy $\cdot$ miRNA $\cdot$ miR-181a $\cdot$ Biomarker $\cdot$ Piglet model $\cdot$ BMP signalling

\section{Introduction}

Highlights

- Altered miRNAs are potential HIE markers, detectable in the first hour of life.

- Altered miRNAs were found consistently in both humans and a porcine model.

- These miRNAs are miRs 374a, 181b, 181a, 151a, 148a and 151a.

- These are correlated with gold standard MRS markers of neonatal encephalopathy.

- Inhibition of miR-181a may confer neuroprotection via the BMP signalling pathway.

- miRNA biomarkers may allow timely diagnosis and therapeutic interventions for HIE

Electronic supplementary material The online version of this article (https://doi.org/10.1007/s12035-020-02018-w) contains supplementary material, which is available to authorized users.

Sophie Casey

Sophie.casey@umail.ucc.ie

Extended author information available on the last page of the article
Neonatal hypoxic ischemic encephalopathy (HIE) is an acute brain injury caused by a hypoxic ischemic insult (HI) due to the disruption of cerebral blood flow and oxygenation [1]. It is one of the most common causes of neonatal death and the most frequent cause of acquired infant brain injury, accounting for approx. 1.5 out of every 1000 live births [2]. It may result from placental abruption, uterine rupture, placenta previa, maternal hypotension, obstructed labour or umbilical cord prolapse [1,3].

The methods currently used to diagnose HIE are inaccurate, and there is an urgent need for reliable and rapidly quantifiable biomarkers of HIE severity $[4,5]$. HIE is a rapidly evolving injury, with initial cell death occurring within minutes of the HI [6], and secondary injury following an interval of reperfusion, occurring approximately $6-12 \mathrm{~h}$ thereafter $[1,7]$. Therapeutic hypothermia (TH) improves outcome in moderate to severe HIE, but rapid diagnosis and application within $6 \mathrm{~h}$ is required for it to be neuroprotective [5, 8-10]. With currently available diagnostic methods, this therapeutic window is often missed [11]. 
The precise timing of the $\mathrm{HI}$ is very difficult to estimate in human neonates. Animal models are necessary to allow control of the timing and duration of the injury. This in turn allows us to examine the timing of HIE-associated molecular alterations and injury mechanisms $[12,13]$. There is growing recognition that large animal and particularly porcine models are more translational [14-16].

miRNAs are small, ubiquitous, non-coding RNA molecules with a hairpin structure [17], which readily cross the blood brain barrier (BBB) to the area of HI [18]. miRNAs play a role in negative regulation of gene expression via inhibition of target messenger RNA (mRNA) translation. Key roles have been defined for miRNAs in mRNA cleavage, differentiation and proliferation, cell death, developmental timing, cellular physiology and spatial patterning of cell fate $[17,19,20]$. It has been proposed that each type of cell at each stage of development may have unique miRNA profiles which 'micromanage' transcriptomic outputs [17].

Hypoxic events are believed to trigger the production of different miRNAs [21] which cross the BBB and migrate to the area of hypoxic ischemic injury via vectors [18] such as exosomes [22, 23]. These miRNAs can be found in the circulation and may act as biomarkers for disease and disordered states such as HIE depending on their levels of expression [24] or 'signatures' [25]. Dharap et al. noted that multiple miRNAs exhibit rapid temporal alterations in their expression levels after an ischemic insult in the adult rat [26], while Ziu et al. have reported that a hypoxic insult causes temporal alterations in miRNA expression in vitro [27]. Our group, among others, have reported that miRNA profiles are altered in umbilical cord blood in humans following a HI, and may have potential in early detection of HIE [5]. However, little is still known about the timing of these changes, whether these miRNAs are conferring protection or damage, or in fact whether similar profiles will be observed in a piglet model.

In this study, we examined the expression profile of all miRNAs in HIE infant umbilical cord blood, and then investigated the evolution of their expression in the circulation of a clinically relevant porcine model of HIE. Following this, we investigated functional effects of inhibiting miRNA in vitro. This is the first study of its kind to explore the circulating temporal whole blood profile of miRNA alterations in a large in vivo model of HIE with accompanying functional analysis. We have reported that miR-374a, miR-181b, miR-181a, miR151a, miR-148a and miR-128 may be cross-species/bridging early biomarkers of HIE, with miR-374a, miR-181b and miR181a levels indicative of moderate-severe injury specifically. miR-181a inhibition increased neurite length in vitro in two different types of cell culture - the SH-SY5Y cell line and primary cultures of E14 rat midbrain - and increased expression of BMPR2 in differentiating SH-SY5Ys. Therefore, this miRNA may be a potential therapeutic target with neuroprotective effects.

\section{Results}

\section{miRNAs Are Significantly Altered in Neonates Who Sustain a Moderate-Severe HI}

To identify miRNAs of interest which differed between HIE neonates and healthy controls, RNA sequencing was performed (Fig. 1a). Analyses of previously performed microarray data showed altered expression levels in 70 miRNAs in umbilical cord whole blood in infants with moderate and severe HIE compared with controls and this data have been previously reported by our group [5]. The results were taken together with those from RNA-seq to aid in selection of miRNAs of interest.

Fifty-five miRNAs were significantly differentially expressed in the comparison between moderate-severe infants and controls (Fig. 1b and Table 1). There was no significant differential expression of any miRNAs in the moderate-severe vs mild comparison or moderatesevere vs PA. Ninety-six miRNAs were not significantly differentially expressed.

The dynamics of the dataset were revealed by converting a set of observation variables into a set of linearly uncorrelated principal component (PC) values. PCs may reveal relationships between variables. $\mathrm{PC} 1$ represents the largest proportion of variation, with smaller effects seen in subsequent PCs. Samples should ideally cluster to the condition of interest, with outliers also identifiable. If samples cluster to anything besides our condition of interest, i.e., moderate-severe HIE, this should be included in linear modelling. Each dot in Fig. $1 \mathrm{c}$ represents a sample, and these are coloured and labelled according to patient group. There was an obvious clustering of controls and most of the mild HIE samples, with PA samples spread across the two clusters. The moderate-severe infant samples clustered towards the top of the plot.

Count-based miRNA expression data were generated from the RNA-seq data by mapping against human miRBase V21 [28]. One hundred fifty-one miRNAs were selected for inclusion in analyses. The total number of counts per sample mapped to human miRBase V21 after filtering is shown in Fig. $1 \mathrm{~d}$.

A panel of 11 miRNAs were selected for analysis of expression in the piglet model, as they were significantly differentially expressed in the RNA-seq and/or microarray. These included miR-27b, miR-30b, miR-30c, miR-128, miR-140, miR-148a, miR-151a, miR-181a, miR-181b, miR-374a and miR-376a. These were chosen due to a significant fold change in expression seen between HIE and controls in both or either the RNA-seq and microarray, availability of ssc compatible PCR primers and also as the majority of these miRNAs are brain enriched in both humans and pigs-determined via Ensembl [29]. 
Fig. 1 Analysis of miRNAs detected by RNA-seq in neonatal umbilical cord blood samples. a Schematic illustrating the process from obtaining initial samples from umbilical plasma to identification of differentially expressed miRs, followed by eventual examination in the porcine model. b Venn diagram showing that 55 miRNAs are significantly differentially expressed in the comparison between moderate-severe infants and controls. No significant differential expression occurred in of any miRNAs in the moderatesevere vs mild comparison or moderate-severe vs PA. A total of 96 miRNAs were not significantly differentially expressed. c PCA of PC1 vs PC2 and $\mathrm{PC} 2$ vs $\mathrm{PC} 3$ for normalised $\log$-CPM values in the data. Each dot represents one sample. $\mathbf{d}$ Fold change of total counts per sample mapped to human miRBase V21 after filtering out miR-486-5p
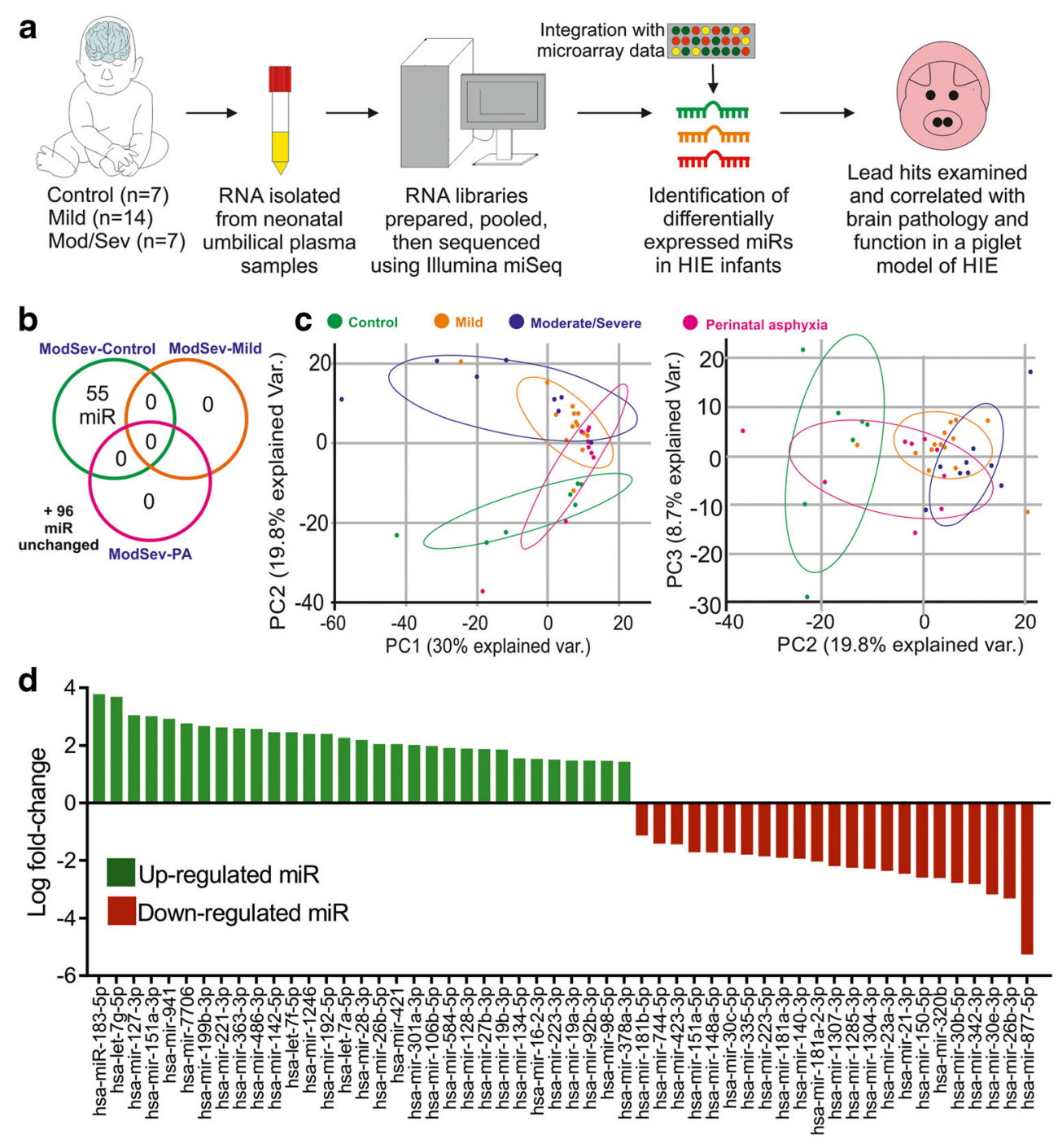

\section{Physiological Parameters of HI in Piglets Reflect Those Observed in Neonates}

Prior to studying miRNA expression in our model organism of interest, we first sought to determine whether the experimental model of HI displayed similarities to the human condition. Utilising the piglet model of HIE, we were able to measure physiological parameters at specific timepoints. Arterial blood gas sampling baseline, during $\mathrm{HI}$ and throughout the postinsult period, was performed in control and $\mathrm{HI}$ animals. Blood physiological parameters included $\mathrm{pH}, \mathrm{pO}_{2}, \mathrm{pCO}_{2}$, $\mathrm{ABE}, \mathrm{HCO}_{3}, \mathrm{Hb}$, Glu, Lac and $\mathrm{iO}_{2} \%$. Mean arterial blood pressure (MABP) was also measured throughout.

Pre-insult, the parameters did not differ significantly between HI and control animals. At the end of the insult, multiple parameters differed significantly in HI compared with controls. MABP was significantly lower when compared with healthy controls $(p=0.001)$. Control piglets MABP remained $>35 \mathrm{mmHg}$, while HI piglets spent an average of $7.95 \mathrm{~min}$ below $35 \mathrm{mmHg}$ and $0.89 \mathrm{~min}$ below $20 \mathrm{mmHg}$. One hour after the insult was terminated, reduced arterial $\mathrm{pH}$ and $\mathrm{ABE}$ and elevated lactate persisted (Table 2).

\section{The Basal Ganglia, Temporal Cortex, Cerebellum and Hippocampus Sustain Injury in the Piglet Model}

To determine whether the physiological changes were also paralleled by brain injury, we performed histopathological examination using H\&E staining of HI piglet brain tissues. This demonstrated that moderate to severe neuronal injury was achieved in five of eight piglets. Figure 2(A)-(H) illustrates the observed cellular differences between control (Fig. 2(A), (C), (E), (G)) and HI (Fig. 2(B), (D), (F), (H)) porcine brains. The hypoxic-ischemic basal ganglia (Fig. 2(B)), temporal cortex (Fig. 2(D)), cerebellum (Fig. 2(F)) and hippocampus (Fig. 2(H)) displayed substantial cellular damage compared with controls (Figs. 2(A), (C), (E), (G)). Two piglets displayed mild damage. One died at 8 -h post-HI 
Table 1 Differential expression analysis showing miRNAs with an adjusted $p$ value $<0.05$ following linear modelling in limma with empirical Bayes moderation. $\log F C \log$ fold change

\begin{tabular}{|c|c|c|c|c|c|}
\hline Upregulated & $\log F C$ & adj.P.Val & Downregulated & $\log F C$ & adj.P.Val \\
\hline hsa-mir-183-5p & 3.782053 & 0.00059 & hsa-mir-181b-5p & -1.12137 & 0.030304 \\
\hline hsa-let-7g-5p & 3.683347 & 0.000147 & hsa-mir-744-5p & -1.40814 & 0.033976 \\
\hline hsa-mir-127-3p & 3.044257 & 0.001585 & hsa-mir-423-3p & -1.43242 & 0.005281 \\
\hline hsa-mir-151a-3p & 3.01409 & 0.000205 & hsa-mir-151a-5p & -1.70168 & 0.003836 \\
\hline hsa-mir-941 & 2.920534 & 0.002135 & hsa-mir-148a-5p & -1.71205 & 0.016131 \\
\hline hsa-mir-7706 & 2.759676 & 0.004994 & hsa-mir-30c-5p & -1.72337 & 0.014088 \\
\hline hsa-mir-199b-3p & 2.670748 & 0.000616 & hsa-mir-335-5p & -1.78559 & 0.014088 \\
\hline hsa-mir-221-3p & 2.621461 & 0.002777 & hsa-mir-223-5p & -1.84476 & 0.01992 \\
\hline hsa-mir-363-3p & 2.584454 & 0.00103 & hsa-mir-181a-3p & -1.89697 & 0.017358 \\
\hline hsa-mir-486-3p & 2.567254 & 0.004722 & hsa-mir-140-3p & -1.9288 & 0.004994 \\
\hline hsa-mir-142-5p & 2.453182 & 0.002543 & hsa-mir-181a-2-3p & -2.0308 & 0.006182 \\
\hline hsa-let-7f-5p & 2.450559 & 0.002593 & hsa-mir-1307-3p & -2.18376 & 0.004357 \\
\hline hsa-mir-1246 & 2.399464 & 0.002515 & hsa-mir-1285-3p & -2.24314 & 0.042551 \\
\hline hsa-mir-192-5p & 2.391835 & 0.001843 & hsa-mir-1304-3p & -2.27537 & 0.013198 \\
\hline hsa-let-7a-5p & 2.261112 & 0.001843 & hsa-mir-23a-3p & -2.35076 & 0.001843 \\
\hline hsa-mir-28-3p & 2.194443 & 0.002828 & hsa-mir-21-3p & -2.45472 & 0.001843 \\
\hline hsa-mir-26b-5p & 2.057607 & 0.003501 & hsa-mir-150-5p & -2.58986 & 0.001997 \\
\hline hsa-mir-421 & 2.05228 & 0.002506 & hsa-mir-320b & -2.60166 & 0.002135 \\
\hline hsa-mir-301a-3p & 2.02168 & 0.009367 & hsa-mir-30b-5p & -2.76987 & 0.00059 \\
\hline hsa-mir-106b-5p & 1.990167 & 0.01992 & hsa-mir-342-3p & -2.81339 & 0.00059 \\
\hline hsa-mir-584-5p & 1.921702 & 0.002593 & hsa-mir-30e-3p & -3.16989 & 0.000465 \\
\hline hsa-mir-128-3p & 1.896204 & 0.004346 & hsa-mir-26b-3p & -3.30217 & 0.000147 \\
\hline hsa-mir-27b-3p & 1.880767 & 0.006182 & hsa-mir-877-5p & -5.25543 & 0.00011 \\
\hline $\begin{array}{l}\text { hsa-mir-19b-3p } \\
\text { hsa-mir-134-5p }\end{array}$ & $\begin{array}{l}1.862158 \\
1.558091\end{array}$ & $\begin{array}{l}0.02526 \\
0.02526\end{array}$ & & & \\
\hline hsa-mir-16-2-3p & 1.535784 & 0.030047 & & & \\
\hline hsa-mir-223-3p & 1.51388 & 0.012056 & & & \\
\hline hsa-mir-19a-3p & 1.483148 & 0.035442 & & & \\
\hline hsa-mir-92b-3p & 1.482061 & 0.016793 & & & \\
\hline hsa-mir-98-5p & 1.472611 & 0.00603 & & & \\
\hline hsa-mir-378a-3p & 1.437922 & 0.005906 & & & \\
\hline hsa-mir-186-5p & 1.175161 & 0.028013 & & & \\
\hline
\end{tabular}

due to cardiac arrest secondary to severe HI and was excluded from further histopathological analysis; another from the HI group was excluded from histopathological analysis due to the entire brain being haemorrhagic. Brain tissue was not collected from another due to coexisting infection.

Using the described scoring system (Total histopathology)( $n$ total brain areas $) /(n$ brain areas scored $)$ [15], we determined that the brains of HI animals were significantly more damaged than those of the controls (Fig. 2(I)) confirming that a moderate-severe injury was achieved $(p=$ $0.01)$. The most prominent areas of injury were the basal ganglia (BG) $(p=0.002)$, hippocampus (Hipp) $(p=0.007)$, frontal cortex (FC) $(p=0.012)$ and temporal lobe (TC) $(p=$ $0.013)$. The cerebellum (CB) $(p=0.069)$ and thalamus $(\mathrm{Th})$
( $p=0.079)$ displayed a milder degree of damage, while the brainstem (BS) $(p=0.221)$ was relatively spared with no significant injury observed compared with controls. No significant neuropathology was noted in the control brain tissues (Fig. 2(J)), in agreement with findings from H\&E staining.

\section{Lac/Naa and Lac/Cr MRS Ratios Are Altered in Response to HI}

To further confirm that piglets had experienced significant $\mathrm{HI}$, in vivo MRS was performed at 70-h post-HI, prior to euthanasia. This demonstrated that metabolite ratios were significantly increased in HI animals for both Lac/NAA $(p=0.02)$ and $\mathrm{Lac} / \mathrm{Cr}(p=0.003)$ when compared with healthy controls 
Table 2 Physiological characteristics of both HI and control piglets. Parameters were measured before the HI, and at the end of the HI, and also $1 \mathrm{~h}$ post-HI. All measures were mean \pm standard deviation or median (IQR). $P$ values were calculated either by independent samples $T$ test or Mann-Whitney $U$ test, depending on distribution of the data. $\mathrm{PO}_{2}$ and $\mathrm{pCO}_{2}$ were measured in $\mathrm{mmHg}, \mathrm{ABE}, \mathrm{HCO}_{3}$, glucose and lactate were measured in $\mathrm{mmol} / \mathrm{L}$ and $\mathrm{Hb}$ was measured in $\mathrm{g} / \mathrm{L}$. $\dagger$ denotes statistical significance between $\mathrm{HI}$ animals and controls

\begin{tabular}{|c|c|c|c|}
\hline & Control & $\mathrm{HI}$ & $p$ value \\
\hline \multicolumn{4}{|l|}{ Pre-insult } \\
\hline $\mathrm{pH}$ & $7.44 \pm 0.05$ & $7.43 \pm 0.03$ & 0.190 \\
\hline $\mathrm{pO}_{2}$ & $90.35(33.68)$ & $85.7(19.13)$ & 0.173 \\
\hline $\mathrm{pCO}_{2}$ & $41.17 \pm 5.28$ & $42.32 \pm 3.37$ & 0.268 \\
\hline $\mathrm{ABE}$ & $2.17 \pm 4.36$ & $3.54 \pm 1.60$ & 0.364 \\
\hline $\mathrm{HCO}_{3}$ & 26.2(1.9) & $27(2.05)$ & 0.066 \\
\hline $\mathrm{Hb}$ & $90.5 \pm 23.63$ & $88.2 \pm 12.56$ & 0.789 \\
\hline Glucose & $6.71 \pm 1.01$ & $12.97 \pm 19.55$ & 0.628 \\
\hline Lactate & $1.42 \pm 0.45$ & $1.89 \pm 1.31$ & 0.413 \\
\hline $\mathrm{iO}_{2} \%$ & $21 \pm 0$ & $21 \pm 0$ & - \\
\hline \multicolumn{4}{|l|}{ End insult } \\
\hline $\mathrm{pH} \dagger$ & $7.46 \pm 0.11$ & $6.90 \pm 0.13$ & $<0.0001$ \\
\hline $\mathrm{pO}_{2} \dagger$ & $96.24 \pm 22.60$ & $26.48 \pm 4.10$ & $<0.0001$ \\
\hline $\mathrm{pCO}_{2}$ & $44.68 \pm 5.09$ & $54.15 \pm 20.23$ & 0.217 \\
\hline $\mathrm{ABE} \dagger$ & $3.97 \pm 2.58$ & $-21 \pm 5.59$ & $<0.0001$ \\
\hline $\mathrm{HCO}_{3} \dagger$ & $27.9 \pm 2.29$ & $7.98 \pm 2.94$ & $<0.0001$ \\
\hline $\mathrm{Hb}$ & $96.87 \pm 12.35$ & $96.9 \pm 16.52$ & 0.995 \\
\hline Glucose $\dagger$ & $6.86 \pm 0.72$ & $9.64 \pm 3.24$ & 0.047 \\
\hline Lactate $\dagger$ & $1.93 \pm 1.03$ & $15.22 \pm 4.21$ & $<0.0001$ \\
\hline $\mathrm{iO}_{2} \% \dagger$ & 21 & $5.5(2.5)$ & $<0.0001$ \\
\hline \multicolumn{4}{|c|}{ 1-h post-insult } \\
\hline $\mathrm{pH} \dagger$ & $7.41 \pm 0.05$ & $7.25 \pm 0.12$ & 0.007 \\
\hline $\mathrm{pO}_{2}$ & $88.45(8.6)$ & $89.3(21.8)$ & 0.625 \\
\hline $\mathrm{PCO}_{2}$ & $47.05 \pm 4.36$ & $43.92 \pm 5.26$ & 0.223 \\
\hline $\mathrm{ABE} \dagger$ & $4.58 \pm 2.27$ & $-7.75 \pm 5.62$ & $<0.0001$ \\
\hline $\mathrm{HCO}_{3} \dagger$ & $28.57 \pm 2.12$ & $18.26 \pm 4.26$ & $<0.0001$ \\
\hline $\mathrm{Hb}$ & $95.5 \pm 13.05$ & $90 \pm 12.73$ & 0.421 \\
\hline Glucose & $6.4 \pm 0.7$ & $6.64 \pm 2.98$ & 0.836 \\
\hline Lactate $\dagger$ & $1.77 \pm 0.88$ & $6.54 \pm 2.8$ & 0.001 \\
\hline $\mathrm{iO}_{2} \%$ & $21(1)$ & $21(5.25)$ & 0.289 \\
\hline
\end{tabular}

(Fig. 2(k)). There was no significant alteration in metabolite ratios for any other measurement (data not shown).

\section{Expression of Six miRNAs Altered $1 \mathrm{~h}$ After $\mathrm{HI}$}

Having validated the model, we next examined whether there were any temporal differences in circulating miRNA expression in HI piglets compared with controls.

Several samples were excluded from the final analysis due to clotting of sample or poor RNA yield, which we set at $<$ $100 \mathrm{ng} / \mu \mathrm{l}$. The final sample numbers for HI animals was $n=$
9 at 0 h, $n=8$ at $1 \mathrm{~h}, n=9$ at $2 \mathrm{~h}, n=8$ at $8 \mathrm{~h}$ and $n=4$ at $72 \mathrm{~h}$. The final sample number for healthy controls was $n=5$ at $0 \mathrm{~h}$, $n=9$ at $1 \mathrm{~h}, n=8$ at $2 \mathrm{~h}, n=6$ at $8 \mathrm{~h}$ and $n=7$ at $72 \mathrm{~h}$. These numbers remained consistent across all miRNAs tested. Of the 11 original miRNAs selected for investigation, six displayed significantly altered expression in a consistent temporal pattern $(p \leq 0.05)$ (Fig. 3).

Two-way ANOVA analysis showed that there was a significant alteration in the expression of miR-148a over time $\left(F_{(4,63)}=15.94 ; p<0.0001\right)$. Post hoc uncorrected Fisher's LSD testing revealed miR-148a was significantly upregulated at $1 \mathrm{~h}$ in $\mathrm{HI}$ animals when compared with healthy timematched controls ( $p=0.0256)$ (Fig. 3a). miR-181b expression was significantly altered over time $\left(F_{(4,63)}=30.44\right.$; $p<0.0001)$ and also due to HI exposure $\left(F_{(1,63)}=7.293\right.$; $p=0.0089)$. Significant upregulation of miR-181b occurred in HI animals at both $1 \mathrm{~h}(p=0.0009)$ and $72 \mathrm{~h}(p=0.0390)$ when compared with controls (Fig. 3b). A relationship between miR-128 expression, exposure to $\mathrm{HI}$ and time $\left(F_{(4,63)}=8.339 ; p<0.0001\right)$ also existed, as well as between miR-128 expression and time alone $\left(F_{(4,63)}=9.58\right.$; $p<0.0001)$. Significant upregulation of miR-128 occurred in $\mathrm{HI}$ animals versus controls at $1 \mathrm{~h}(p=0.0263)$ and at $2 \mathrm{~h}$ $(p<0.0001)$ (Fig. 3c). miR-181a expression was significantly altered over time $(p<0.0001)$, with significant upregulation of miR-181a in HI animals when compared with time-matched controls $(p=0.0414)$ (Fig. 3d). miR-151a expression was significantly altered over time $\left(F_{(4,63)}=33.38 ; p<0.0001\right)$ and also due to HI exposure $\left(F_{(4,63)}=5.148 ; p=0.0267\right)$. Significant upregulation of miR-151a was noted in HI animals when compared with controls at $1 \mathrm{~h}(p=0.006)$ (Fig. 3e). miR-374a expression was significantly altered over time $\left(F_{(4,63)}=10.32 ; p<0.0001\right)$, with a significant upregulation in HI animals when compared with controls at $1 \mathrm{~h}(p=$ 0.0156) (Fig. 3f).

miR-27b, miR-30b, miR-30c and miR-140 all exhibited a significant relationship between miR expression and time $(p<0.001)$ using two-way ANOVA, but post hoc Fisher's LSD testing did not reveal any differences between timematched HI samples and controls. All showed an increase in expression at $1 \mathrm{~h}$ in $\mathrm{HI}$ animals when compared with controls, but none reached statistical significance (Supplementary Fig. 1a-d). miR-376a was excluded from the analysis as there was no sequence homology between the human and porcine gene (Table 5). In keeping with this, CT values for miR-376a were high, indicating late amplification and low/no abundance of the miRNA in the sample.

When observing controls, a slight natural fluctuation in miRNA expression could be observed over time. Expression levels of the miRNAs generally appeared to rise and then fall in a temporal manner. Applying a HI disturbed this flux and caused expression levels to rise rapidly to peak at approximately $1 \mathrm{~h}$ after the insult was terminated (in contrast, levels 

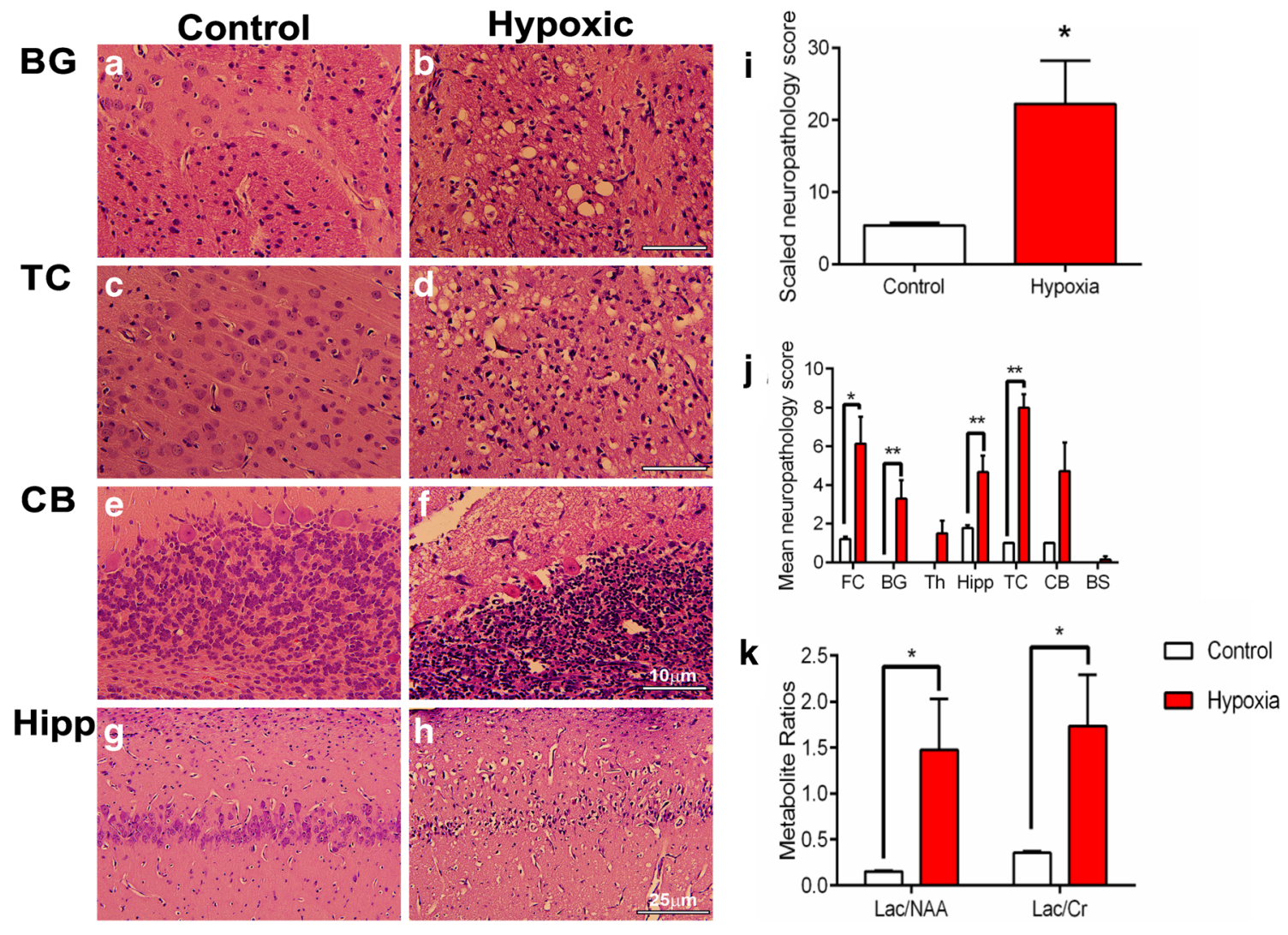

Fig. 2 Hypoxic Ischemic exposure leads to neuropathological changes and increases in cerebral metabolite ratios. (A-H) H\&E staining of $n=10$ controls (A, C, E, G) versus $n=7 \mathrm{HI}(\mathrm{B}, \mathrm{D}, \mathrm{F}, \mathrm{H})$ porcine brains illustrating increased cellular neuronal injury $(\mathrm{A}, \mathrm{B})$ represent the basal ganglia $(\mathrm{BG}),(\mathrm{C}, \mathrm{D})$ represent the temporal cortex $(\mathrm{TC}),(\mathrm{E}, \mathrm{F})$ represent the cerebellum $(\mathrm{CB})$ and $(\mathrm{G}, \mathrm{H})$ represent the hippocampus (Hipp). A-F are $\times 40$ images, with $10-\mu \mathrm{m}$ scale bars. $(\mathrm{G}, \mathrm{H}) \times 20$ images, with $25-\mu \mathrm{m}$

of miR-128 dramatically drop at $2 \mathrm{~h}$ ). Expression levels for all aforementioned miRNAs decreased over time and were no longer different from controls from 8-h post-HI onwards.

Animals which had histopathological outcome data were separated by into mild $\mathrm{HI}$ and moderate-severe $\mathrm{HI}$ groups to allow examination of whether miRNA expression alterations were associated with pathological outcome. Two-way ANOVA analysis showed that there was a significant alteration in miR-181b over time $\left(F_{(4,50)}=17.27 ; p<0.0001\right)$, with expression in moderate-severe animals significantly higher than controls at $1 \mathrm{~h}(p=0.0033)$ and $72 \mathrm{~h}(p=0.0283)$. This difference at $1 \mathrm{~h}$ also persisted following Bonferroni correction $(p=0.0098)$ (Fig. 4a). miR-181a expression was also significantly altered over time $\left(F_{(4,48)}=13.5\right.$; $p<0.0001)$, with this upregulation occurring in moderate-severe animals at $1 \mathrm{~h}(p=0.0415)$ (Fig. $4 \mathrm{~b})$. miR-374a expression was significantly altered over time $\left(F_{(4,50)}=3.28 ; p=0.0183\right)$, with expression in moderatesevere animals significantly higher than that seen in controls at $1 \mathrm{~h}(p=0.024)$ (Fig. $4 \mathrm{c})$. scale bars. (I, J) Histogram showing mean neuropathology in (I) the overall scaled neuropathology score between the two groups and $(\mathrm{J})$ individual brain regions. (K) Histogram of magnetic resonance spectroscopy (MRS) data of metabolite ratios in $n=9$ controls versus $n=5 \mathrm{HI}$ animals for both Lac/NAA and $\mathrm{Lac} / \mathrm{Cr}$. All data are mean \pm SEM $(*=p \leq 0.05, * *=p \leq 0.01, * * *=p \leq 0.001$; Mann-Whitney $U$ tests)

\section{Lac/Cr Ratio and Differentially Expressed miRNAs Were Positively Correlated}

We sought to investigate whether there was any relationship between altered miRNA expression at $1 \mathrm{~h}$, MRS ratios and histology scores. Supplementary Table 2 illustrates a Spearman's correlation table across 15 variables with all values represented by $(r s)$. Lac/Cr ratio was correlated positively and significantly with five out of six differentially expressed miRNAs at $1 \mathrm{~h}$. Positive correlations could be seen with scaled histology score $(r s=0.602 ; p=0.023)$, miR-374a $(r s=0.624 ; p=0.023)$, miR-181b $(r s=0.856 ; p<0.0001)$, miR-128 ( $r s=0.575 ; p=0.04), \operatorname{miR}-151 \mathrm{a}(r s=0.729 ; p=$ $0.005)$ and miR-181a $(r s=0.615 ; p=0.033)$. The strongest positive correlation with $\mathrm{Lac} / \mathrm{Cr}$ was observed with miR181b. Lac/NAA was positively and significantly correlated with scaled histology score $(r s=0.552 ; p=0.041)$, miR$181 \mathrm{~b}(r s=0.593 ; p=0.033)$ and miR-181a $(r s=0.587 ; p=$ 0.045).

Linear regressions were performed to determine whether miRNAs of interest have predictive value based on 
Fig. 3 His 1tograms representing $\log _{10} 2^{-} \Delta \Delta C$ T of significantly altered miRNAs a miR-148a, b miR-181b, c miR-128, d miR181a, e miR-151a and f miR-374a at $0 \mathrm{~h}, 1 \mathrm{~h}, 2 \mathrm{~h}, 8 \mathrm{~h}$ and $72 \mathrm{~h}$ in controls and post-HI with evolution over time. All data are mean \pm SEM; two-way ANOVA with post hoc uncorrected Fishers LSD test. A single asterisk indicates statistical significance of between cases and time-matched control samples, $* *=p \leq 0.01$, $* * *=p \leq 0.001$
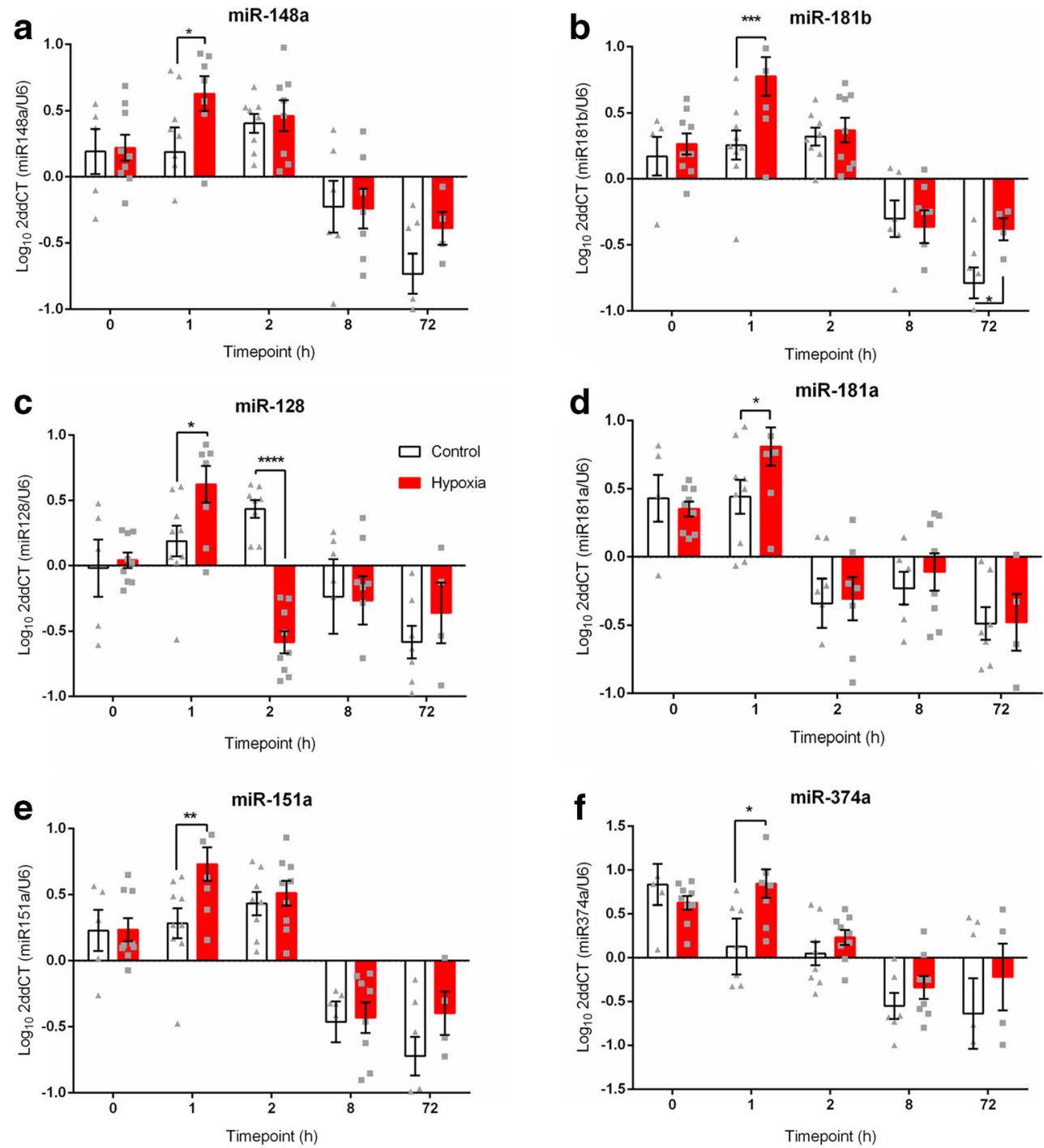

histopathological outcome and common MRS markers (Table 3). Histopathological outcome was associated with miR-374a alterations $F_{(1,15)}=7.875 ; p=0.01 ; \mathrm{R} 2=0.344$, miR-181b alterations $F_{(1,15)}=17.719 ; p=0.001 ; R^{2}=0.542$, miR-128 alterations $F_{(1,15)}=17.175 ; p=0.001 ; R^{2}=0.534$, miR-151a alterations $F_{(1,15)}=17.906 ; p=0.001 ; R^{2}=0.544$, miR-181a alterations $F_{(1,15)}=14.539 ; p=0.002 ; R^{2}=0.492$; $\mathrm{Lac} / \mathrm{Cr} F_{(1,13)}=769.621 ; p<0.0001 ; R^{2}=0.983$ and Lac/ NAA $F_{(1,13)}=183.998 ; p<0.0001 ; R^{2}=0.934$ and vice versa. miR-374a expression at $1 \mathrm{~h}$ was associated with $\mathrm{Lac} / \mathrm{Cr}$ $F_{(1,11)}=5.25 ; p=0.043 ; R^{2}=0.323$ and Lac/NAA $F_{(1,11)}=$ $5.393 ; p=0.04 ; R^{2}=0.329$. miR-181b expression at $1 \mathrm{~h}$ was associated with $\mathrm{Lac} / \mathrm{Cr} F_{(1,11)}=31.839 ; p<0.0001 ; R^{2}=$ 0.743 and Lac/NAA $F_{(1,11)}=26.383 ; p<0.0001 ; R^{2}=0.706$. miR-128 expression was associated with $\mathrm{Lac} / \mathrm{Cr} F_{(1,11)}=$ $21.92 ; p=0.001 ; R^{2}=0.666$ and Lac/NAA $F_{(1,11)}=19.9$; $p=0.001 ; R^{2}=0.644$. miR-151a expression was associated with $\mathrm{Lac} / \mathrm{Cr} F_{(1,11)}=20.488 ; p=0.001 ; R^{2}=0.651$ and Lac/ NAA $F_{(1,11)}=16.788 ; p=0.002 ; R^{2}=0.604$. miR-148a expression was associated with $\mathrm{Lac} / \mathrm{Cr} F_{(1,10)}=6.309 ; p=$ $0.031 ; R^{2}=0.387$ and Lac/NAA $F_{(1,10)}=5.436 ; p=0.042$; $R^{2}=0.352$. miR-181a expression was associated with Lac/ Cr $F_{(1,11)}=21.113 ; p=0.001 ; R^{2}=0.657$ and Lac $/ \mathrm{NAA}$ $F_{(1,11)}=15.743 ; p=0.002 ; R^{2}=0.589$.

\section{Predicted miRNA Targets Play Roles in Vital Signalling Cascades, Biological Processes and Molecular Functions}

We investigated target genes of each of the six significantly altered miRNAs and aimed to determine which genes, if any, were common between miRNAs. A total of 1987 genes were predicted to be targets of the miRNAs with high confidence. miR-181b had 705 targets, miR-151a had 27, miR-128 had 465, miR-374a had 502, miR-181a had 12 and miR-148a had 276. Out of these, targeting of one gene, FKBP1A, was shared among four miRNAs - miR-148a, miR-151a, miR-181b and miR-374a. Figure 5 illustrates miRNAs which share common 
(a)

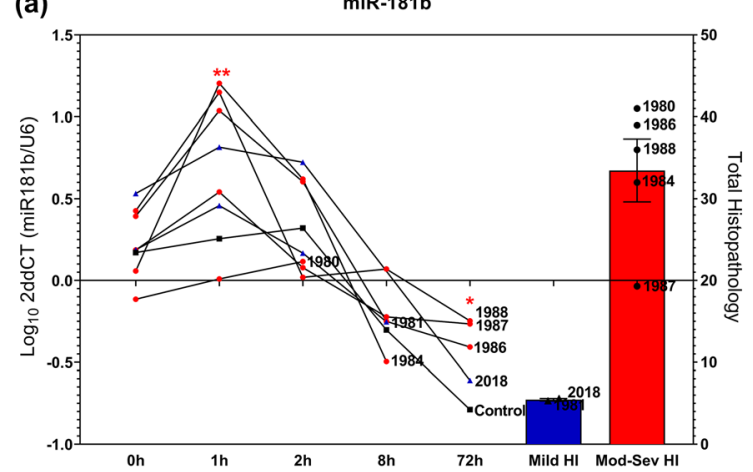

(c)

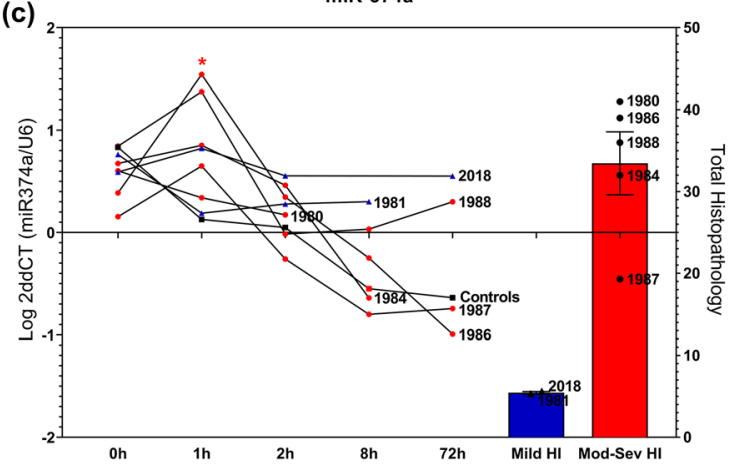

Fig. 4 Temporal evolution of miRNA expression in control, mild and moderate-severe piglets alongside histopathology a miR-181b, b miR$181 \mathrm{a}$ and $\mathbf{c}$ miR-374a, at $0 \mathrm{~h}, 1 \mathrm{~h}, 2 \mathrm{~h}, 8 \mathrm{~h}$ and $72 \mathrm{~h}$ in controls and post-HI with evolution over time. Histopathological scores are shown as bar graphs. All data are mean \pm SEM; two-way ANOVA with post-hoc

targets. A more detailed list of the target genes and corresponding miRNAs is outlined in Supplementary Table 2.

Using EnrichR, it was determined that genes targeted by miR-148a appeared to be most highly abundant in the colon $(p=0.00323)$, those targeted by miR-181b were most abundant in the amygdala $(p=0.002746)$, miR-128 targets were most abundant in adipocytes $(p=0.009988)$, miR-181a targets were most abundant in CD56+ natural killer (NK) cells $(p=0.009033)$, miR-151a targets were most abundantly
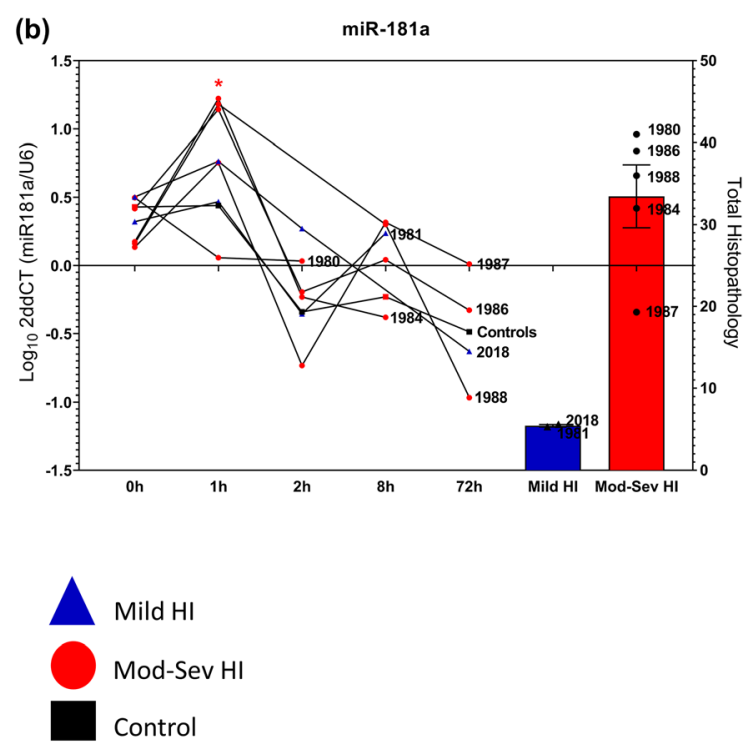

uncorrected Fishers LSD test. A single asterisk indicates statistical significance of $p \leq 0.05$ between cases and time-matched control samples, $* *=p \leq 0.01, * * *=p \leq 0.001$. Red stars indicate statistical significance between mod-sev HI vs control

expressed in the superior cervical ganglion $(p=0.04647)$ and miR-374a targets were most abundant in the pineal gland $(p=0.01680)$.

KEGG pathway analysis was performed for targets of each of the six miRNAs (Fig. 6). All six miRNAs shared two common KEGG pathways - the MAPK signalling pathway and the neurotrophin signalling pathway. Regarding the MAPK signalling pathway, the most significantly associated miRNAs were miR-374a $(p=0.0013)$

Table 3 Linear regression $\left(R^{2}\right)$ of MRS variables, scaled histology score and significant miRNAs expression levels at 1-h post-HI. All values are coefficient of determination $\left(R^{2}\right)$. Italicised values indicates statistical significance; $(p=0.05)$

\begin{tabular}{|c|c|c|c|c|c|c|c|c|c|}
\hline & Scaled Histology & miR-374a & miR-181b & miR-128 & miR-151a & miR-148a & miR-181a & $\mathrm{Lac} / \mathrm{Cr}$ & $\mathrm{Lac} / \mathrm{Naa}$ \\
\hline Scaled Histology & 1 & & & & & & & & \\
\hline miR-374a & 0.344 & 1 & & & & & & & \\
\hline miR-181b & 0.542 & 0.668 & 1 & & & & & & \\
\hline $\operatorname{miR}-128$ & 0.534 & 0.724 & 0.914 & 1 & & & & & \\
\hline miR-151a & 0.544 & 0.698 & 0.965 & 0.938 & 1 & & & & \\
\hline miR-148a & 0.222 & 0.727 & 0.796 & 0.8 & 0.782 & 1 & & & \\
\hline miR-181a & 0.492 & 0.584 & 0.92 & 0.79 & 0.877 & 0.705 & 1 & & \\
\hline $\mathrm{Lac} / \mathrm{Cr}$ & 0.983 & 0.323 & 0.743 & 0.666 & 0.651 & 0.387 & 0.657 & 1 & \\
\hline $\mathrm{Lac} / \mathrm{NAA}$ & 0.934 & 0.329 & 0.706 & 0.644 & 0.604 & 0.352 & 0.589 & 0.971 & 1 \\
\hline
\end{tabular}




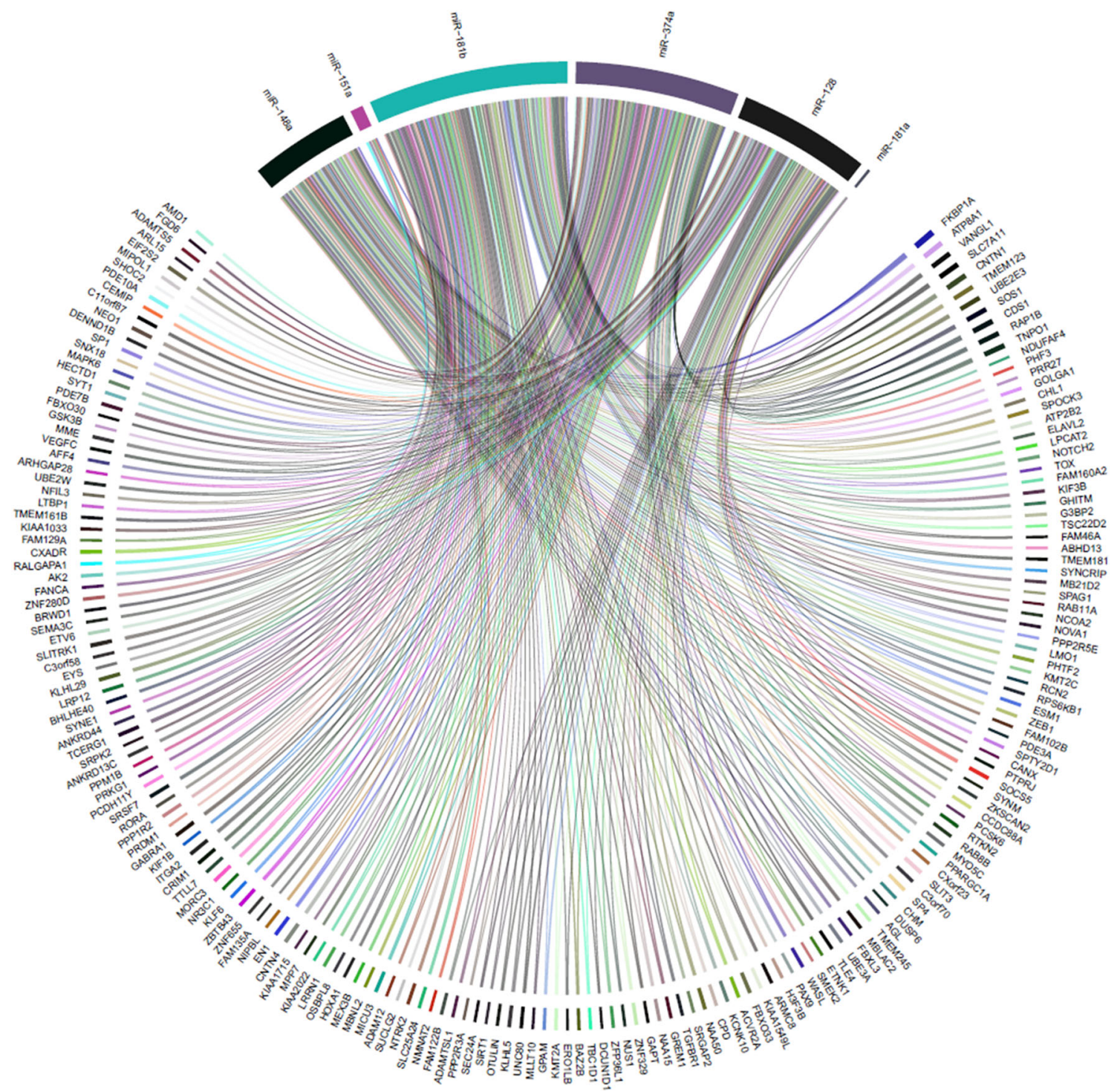

Fig. 5 Predicted targets regulated by early miRNA biomarkers of $\mathrm{HI}$ in the porcine model. Chord diagram illustrating predicted targets of each significantly altered miRNA and those which were found to be common

(Fig. 6f) and miR-128 ( $p=0.02119$ ) (Fig. 6a). Less significantly associated miRNAs included miR-148a $(p=$ $0.7742)$, miR-181b $(p=0.1004), \operatorname{miR}-181 \mathrm{a}(p=0.1634)$ and miR-151a $(p=0.3307)$. Within the MAPK signalling pathway, miR-128, miR-181b and miR-374a commonly targeted RAP1B and SOS1 (Fig. 6a-f). Regarding the neurotrophin signalling pathway, the most significantly associated miRNAs included miR-128 ( $p=0.0067)$ (Fig. 6a) and miR-181b ( $p=0.0094)$ (Fig. 6e). Less significantly associated miRNAs included miR-181a $(p=0.06913)$, miR-151a $(p=0.1489)$, miR-374a $(p=0.1798)$ and miR148a $(p=0.8084)$. Within the neurotrophin signalling pathway, miR-128, miR-181b and miR-374a commonly targeted RAP1B and SOS1, identical to the MAPK signalling pathway. The most commonly associated genes of each KEGG pathway are illustrated in Fig. 6. between them. A detailed insight into the predicted downstream targets of each gene and those which were shared between miRNAs is outlined in Supplementary Table 2

Gene ontologies of the lists of target genes were examined in terms of biological processes (Fig. 6) and we elucidated that all six miRNAs were associated with the following 22 biological processes - regulation of transcription from RNA polymerase II promoter; T cell activation; regulation of BMP signalling pathway; regulation of apoptotic process; positive regulation of transcription, DNA templated; regulation of transmembrane receptor protein serine/threonine kinase signalling pathway; non-canonical Wnt signalling pathway; activation of protein kinase activity; regulation of transcription, DNA templated; positive regulation of gene expression; organic anion transport; CNS development; protein maturation; protein processing; positive regulation of apoptotic process; regulation of signal transduction; protein phosphorylation; positive regulation of nucleic acid-templated transcription; negative regulation of gene expression; and proteolysis. 

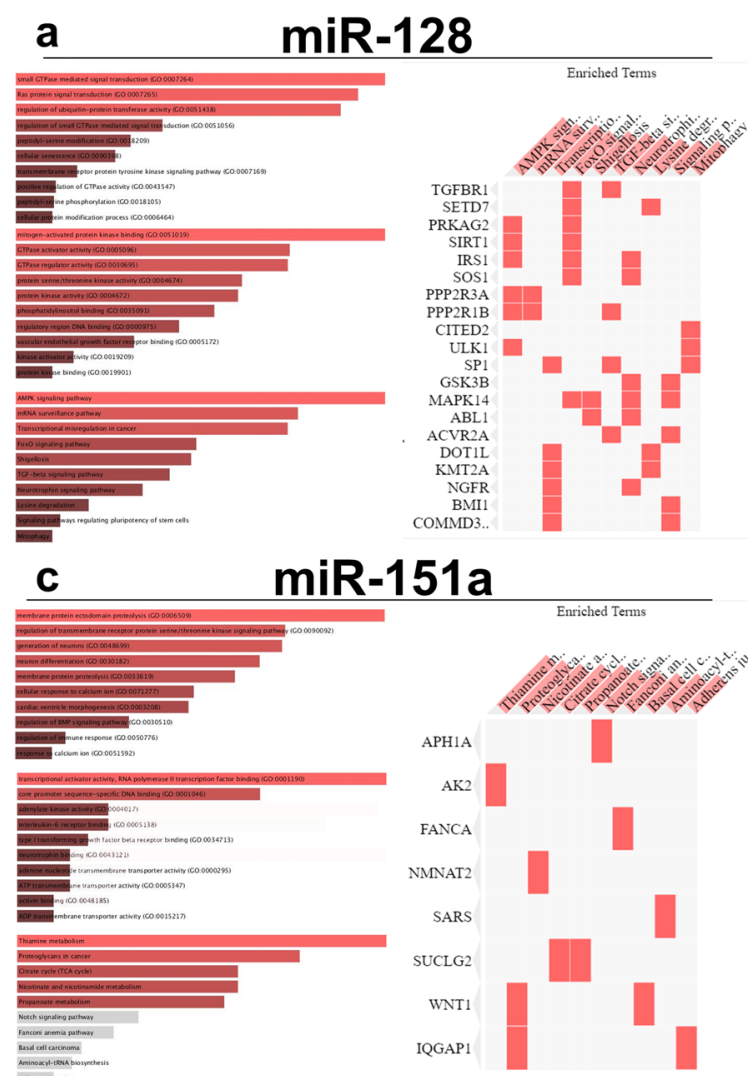

\section{e}

miR-181b
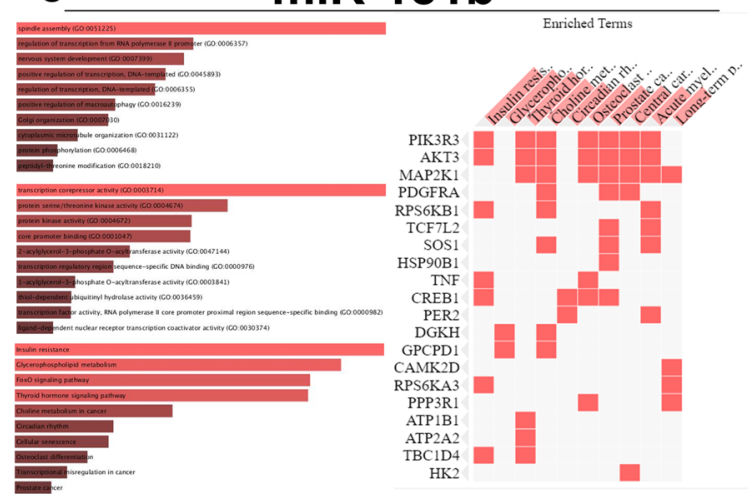

Fig. 6 EnrichR pathway analysis of the predicted targets of a miR-128, $\mathbf{b}$ miR-148a, c miR-151a, d miR-181a, e miR-181b and f miR-374a. Anticlockwise groups of images represent the most significantly associated biological processes, the most significantly associated

\section{Inhibition of miR-181a Increases BMPR2 Expression and Neurite Length In Vitro}

Finally, immunocytochemistry was performed to determine whether inhibition of miR-181a affected expression of members of the BMP signalling pathway or neuronal growth in vitro. Figure 7 depicts this in healthy control and miR181a inhibited cells.

Inhibition of miR-181a increased expression of BMPR2 in SH-SY5Y cells $(p<0.0001)$ (Fig. 7a). Since increased BMP expression has also been associated with increased axonal
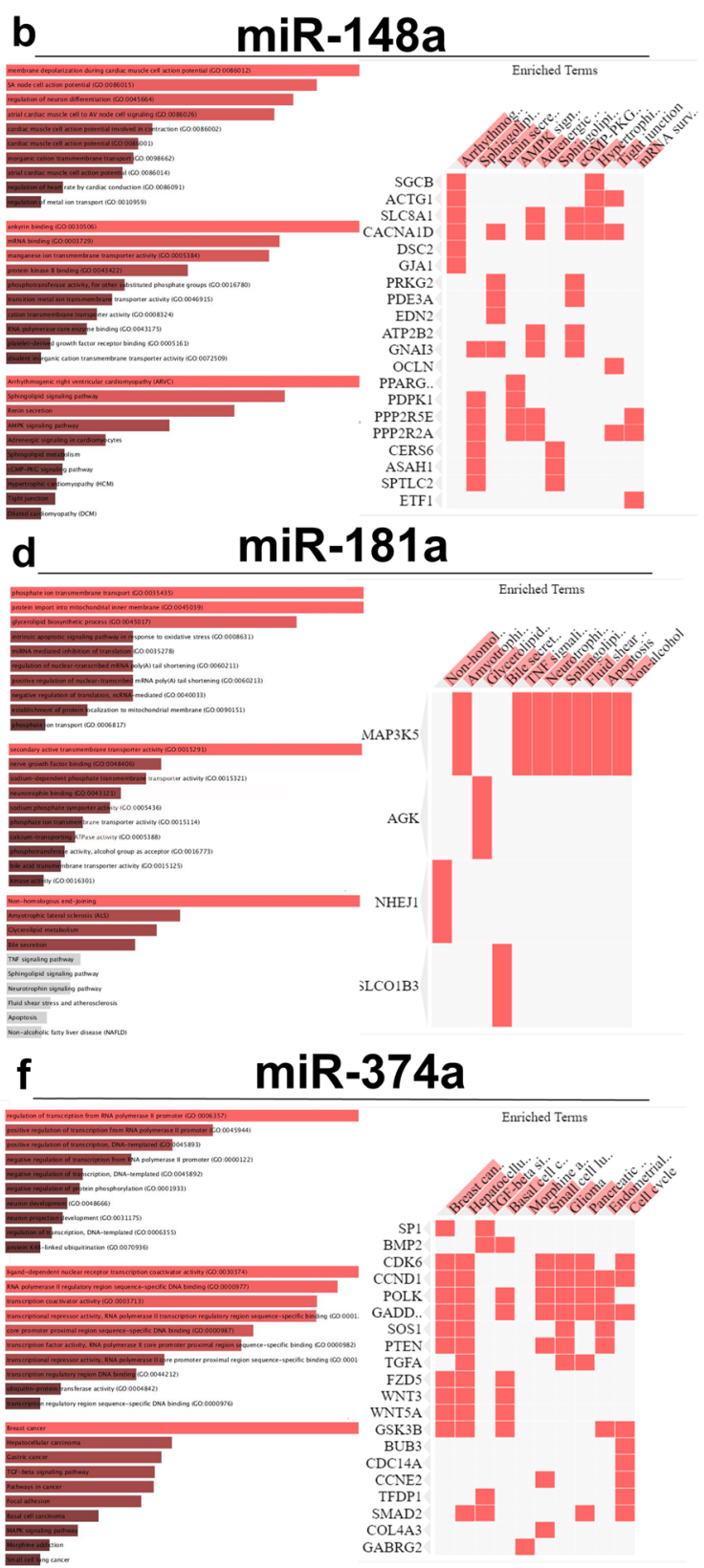

molecular functions, the most significantly associated KEGG pathways and the most significantly associated genes in the aforementioned KEGG pathways. All are ranked by $p$ value

growth under certain conditions [30], neurite length was measured. Inhibition of miR-181a increased neurite length after $24 \mathrm{~h}$ in SH-SY5Y cells $(p=0.0225)$ (Fig. 7b, c), which prompted a longer-term examination of the effect of miR181a inhibition in primary cultures of E14 rat midbrain. Neurite length was increased in response to both time $\left(F_{(1,4)}=25.32 ; p=0.0073\right)$ and treatment with the miR-181a inhibitor $\left(F_{(1,4)}=9.002 ; p=0.009\right)$. Bonferroni's test for multiple comparisons revealed that neurite length was significantly increased in primary cultures after $72 \mathrm{~h}(p=0.0076)$ (Fig. $7 \mathrm{~d}, \mathrm{e})$. 

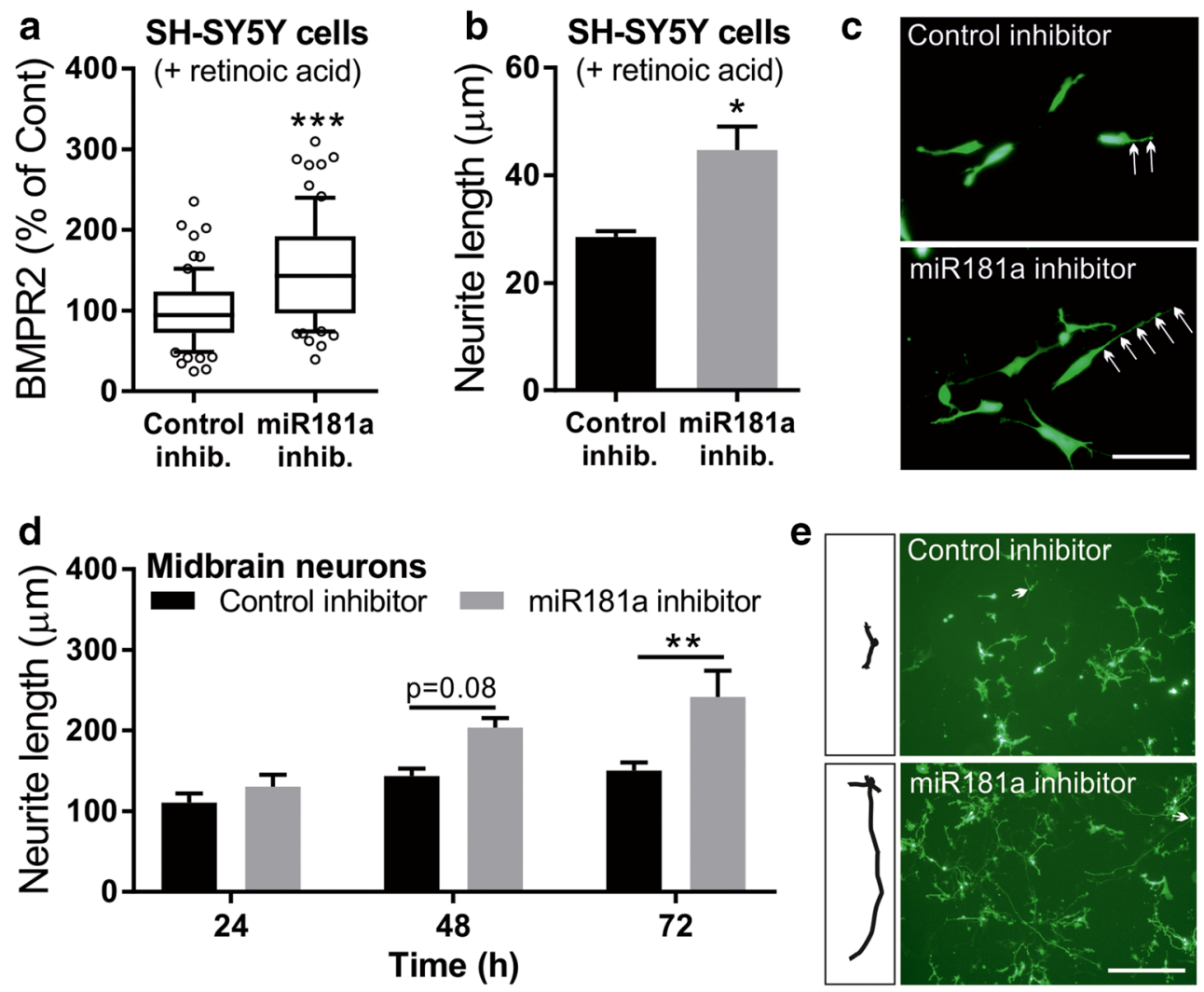

Fig. 7 Inhibition of miR-181a modulates target gene expression and promotes neurite growth in SH-SY5Y cells and in primary cultures of rat midbrain neurons. a Box and whisker plot showing relative levels of BMPR2 expression in SH-SY5Y cells at 24-h post-transfection with $500 \mathrm{nM}$ of a control or miR-181a inhibitor with a GFP-expressing plasmid to identify transfected cells. Data are shown in boxplots as the 10-90th percentile with outliers shown as individual datapoints of $n=74$ individual cells taken from three independent experiments $(* * * p<0.001$, Students $t$ test). Graph (b) and photomicrograph (c) represent neurite length of SH-SY5Y cells grown in the presence of $20 \mu \mathrm{M}$ retinoic acid

(RA) for 24-h post-transfection with $10 \mu \mathrm{l}$ of control or miR-181a inhibitor with a GFP-expressing plasmid. Data are all \pm SEM of three independent experiments $(* p<0.05$, Students $t$ test). Graph (d) and photomicrograph/neuronal tracings (e) represent neurite length of primary cultures of embryonic day 14 rat midbrain neurons at 24-, 48and 72-h post-transfection with $500 \mathrm{nM}$ control or miR-181a inhibitor with a GFP-expressing plasmid. Data are all mean \pm SEM of three independent experiments $(* * p<0.05$, two-way ANOVA with post hoc Bonferroni test)

\section{Discussion}

Molecular biomarkers of HIE are required to support diagnosis and inform treatment decisions. Here, we combined miRNA profiling of human umbilical cord blood following HIE with a targeted approach in a HI porcine model to identify six miRNAs-miR-128 miR-148a, miR-151a, miR-181a, miR-181b and miR-374a — whose expression is significantly, consistently and rapidly upregulated at an early time point1-h post-HI, with equally rapid resolution. Three miRNAs were specifically upregulated in moderate-severe $\mathrm{HI}-\mathrm{miR}$ 374a, miR-181a and miR-181b. We have demonstrated correlations between five miRNAs and $\mathrm{Lac} / \mathrm{Cr}$ ratios from in vivo MRS of the same cohort, suggesting a direct association with neuronal injury. Inhibition of miR-181a was examined in vitro and increased neurite length in both SH-SY5Y cells and primary cultures of rat midbrain neurons. The predicted downstream targets of the six miRNAs were predicted to exert neuroprotective effects, and functional examination of one

of the associated pathways - the BMP signalling pathway (a member of the TGF $\beta$ superfamily [31])—in differentiating SH-SY5Y cells revealed inhibition of miR-181a increases expression of the type II receptor BMPR2.

Our findings support the existence of circulating miRNA biomarkers of HIE linked to clinically measured outcomes that may support diagnostic/therapeutic decision making as well as potentially allowing rapid identification of severely injured neonates. Furthermore, therapeutic inhibition of miR-181a may promote the vital functions of the BMP signalling pathway in neonates - i.e. osteogenesis, embryogenesis, tissue homeostasis and neurogenesis. Our findings are in agreement with many miRNA-HIE studies in which miRNAs are upregulated in response to HIE $[32,33]$ and inhibition provides protection $[25,32,34]$. However, more in-depth research with larger sample size is needed on miRNAs as therapeutic agents and their effects in response to $\mathrm{HI}$ in vitro and in vivo. Due to the rapidly evolving nature of HIE [6] and slow diagnostic methods which result in the therapeutic 
window being missed [11], there is an urgent need for rapidly, easily accessible biomarkers of HIE. We believe the aforementioned six miRNAs are clinically relevant, cross-species biomarkers of HIE, particularly moderate-severe injury.

This is, as far as we know, the first study to show miR128 's association with hypoxia and HIE. However, other studies have indirectly alluded to this link via its role in modulation of reactive oxygen species (ROS) in areas such as bronchial epithelial and medulloblastoma cells [35]. ROS play a key role in HIE, rapidly increasing in number and ultimately resulting in inflammation, damaging membranes, lipids, DNA and proteins [36]. This miRNA is important for CNS development and physiological homeostasis, with involvement in proliferation, differentiation and apoptosis. Expression patterns are largely tissue specific and developmentally regulated. Circulating miR-128 expression is altered in some cancers [37], for example, in neuroblastoma, glioblastoma, prostate cancer, lung cancer and leukaemia, making it a good biomarker candidate for many diseases and disorders [38-42]. Upregulation is noted in another disorder of the perinatal period-preeclampsia [43].

miR-151a dysregulation has previously been linked to intracellular hypoxia $[35,44,45]$. miR-151a is thought to play an oncogenic role in certain cancers by increasing cell invasion and migration [46]. It may also play a key role in regulation of cellular respiration and production of ATP via targeting of cytochrome B during mitochondrial dysfunction [47]. Altered expression of miR-151a has also been linked with schizophrenia [48] and Parkinson's disease [49].

We consider miR-181b to be the most promising bridging biomarker candidate out of the six. Previous findings from our group suggest that miR-181b expression is dysregulated in the umbilical cord blood of moderate-severe HIE neonates [50, 51], as reflected in our moderate-severe porcine cohort. miR$181 \mathrm{~b}$ is abundant in the brain, and is one of the most widely studied miRNAs as expression is dysregulated in tumour pathogenesis [52]. Downregulation in brain tissue of a mouse model of ischemia up to $24 \mathrm{~h}$ after injury has also been noted [53]. Downregulation of miR-181b has proven neuroprotective in mouse brain tissue [54] as well as providing cellular protection in thyroid cancer [55]. To determine whether the effect of miR-181b upregulation in response to HI has neuroprotective or detrimental effects, further investigation into its downstream targets and their effects is required. One predicted downstream target is ubiquitin C-terminal hydrolase L1 (UCHL-1), a proposed biomarker of HIE and traumatic brain injury. Alterations in cord UCHL-1 have been reported in neonatal HIE previously [1].

We consider miR-374a to be another promising bridging biomarker candidate for neonatal HIE, when considering this study alongside previous studies in umbilical cord blood of HIE neonates by our group and others $[5,51,56]$. It may be particularly useful in identification of moderate-severe cases.
Another study of human HIE subjects has also noted a downregulation of miR-374a [56]. The one previous piglet model study of the effects of hypoxia on miRNA also reported an upregulation of miR-374a expression in piglet plasma samples as soon as $30 \mathrm{~min}$ after $\mathrm{HI}$ with rapid recovery thereafter [57], increasing confidence in our results. We have proven that expression of circulating miR-374a is rapidly and significantly altered in both the umbilical blood of neonates and arterial blood of piglets after HI when compared with controls, with rapid decrease in expression variability and downregulation at the time of insult. Expression is increased 1-h post-insult and returns to control levels by 8 -h post-insult. Further examination is required to elucidate the functional effects of miR-374a inhibition in response to $\mathrm{HI}$ and neuronal recovery.

miR-181a has not, to the best of our knowledge, been examined in hypoxic ischemic encephalopathy previously. The present study has identified alterations in the expression of this miRNA in neonatal HIE whole blood and plasma samples, as well as HI porcine whole blood, particularly moderate-severe HI. Due to the novelty of this finding, we sought to determine its functional effects in vitro, in healthy cells. Inhibition in healthy cells increased expression of BMPR2 in differentiating SH-SY5Y cells, and increased neurite growth in two separate cell lines. Little is currently known about the effects of miRNAs in the HIE brain [58]. Whether this miRNA-driven increase in neurite length is neuroprotective, or may interfere with neuronal survival is unclear, as existing studies are conflicting. miR-181a overexpression may play a detrimental, damaging role in HIE, as inhibition promotes neurite growth, which is typically associated with neuroprotection $[59,60]$; but on the other hand, it may exert aberrant effects during HIE [61]. Further research using miR-181a mimics and is required to further elucidate its role. miR-181a has been reported as a key posttranscriptional modulator of $\mathrm{T}$ cell receptor signalling strength/sensitivity; abundantly expressed in immature $\mathrm{T}$ cells and greatly reduced in more mature $\mathrm{T}$ cells [62]. Like many other miRNAs, miR-181a has been cited as a potential biomarker for several diseased and disordered states. In the context of HIE, levels of this miRNA were raised within $1 \mathrm{~h}$ of cerebral ischemia in the rat hippocampus [63]. Kong et al. state that miR-181a may act neuroprotectively on microglia in early HI [64], in line with the protective roles of the predicted targets of our miRNAs of interest. Inhibition of this miRNA in healthy cells increased BMP signalling - vital for osteogenesis, postnatal skeletal homeostasis [65, 66], cardiogenesis [67], embryogenesis [68], neurogenesis [69] and organogenesis [70]. BMP mutations result in deleterious effects such as embryonic lethality [71] and skeletal disorders [66]. Samanta et al. demonstrated that administration of BMP antagonist noggin provides neuroprotection against ischemic brain injury in mice [72]. BMP2 overexpression reportedly provides protection against deleterious apoptotic processes, and is believed to act via BMPR2 [73]. Inhibition of miR- 
181a may therefore exert neurotrophic effects and provide neuroprotection via similar mechanisms.

miR-148a was previously found to be altered between HIE and control groups in a microarray performed by our group [5], and is reported as a 'hypoxamir' by Nallamshetty et al., upregulated in response to HI [35], which coincides with the findings outlined in this study. This miRNA may prompt apoptosis under certain conditions, supporting the notion that miRNAs are key regulators of apoptosis [74].

Some predicted targets of the miRNAs of interest have been implicated in HIE previously, but simultaneously, many lack research in the area. FKBP1A was linked to several differentially expressed miRNAs of interest and has been investigated in $\mathrm{HI}$ in a rat model [75]. FKBP1A is a $\mathrm{T}$ cell binding protein with roles ranging from immunoregulation to protein trafficking [76], and is recruited late in the hypoxia signalling cascade [77]. The MAPK signalling pathway, associated with each miRNA of interest reportedly contributes to antiinflammatory effects exerted by neuroprotective BDNF in response to a HI [78]. The neurotrophin signalling pathway has also been linked to each of the miRNAs of interest and implicated in HI. Expression of neurotrophins 3 and 4 is essential in early neurodevelopment, and levels are reportedly reduced in response to HI [79]. It remains unclear whether miRNAs linked to the above signalling pathways and target genes provide neuroprotection after the spike in expression we see at $1 \mathrm{~h}$, although many are reported to. Further investigation into mechanisms of action in healthy and hypoxic brains is required.

A major advantage of this study is that neurodevelopment of the porcine model is very similar to that of the human brain at birth [80], increasing the likelihood that this research is translatable to humans. We observe similar patterns of injury in porcine histopathology as we do in human cases of HIE [15, $81,82]$ - the cortex is the most prominently injured area, as well as the basal ganglia. We have also observed MRS ratios of Lac/NAA and $\mathrm{Lac} / \mathrm{Cr}$ in our piglet model which reflect the human condition. $\mathrm{Lac} / \mathrm{Cr}$ and $\mathrm{Lac} / \mathrm{NAA}$ are potentially the best quantitative MRS biomarker of neurodevelopmental outcome after HIE [83-86]. This model is therefore clinically relevant, and the results have translational significance. We have analysed the temporal profiles of a wide range of miRNAs using qRT-PCR and gained novel insight into expression profiles of each of these under normal circumstances and after $\mathrm{HI}$ over $72 \mathrm{~h}$. Another asset to this study is that the altered expression of these miRNAs is consistent with that seen in our biomarker discovery methods utilising human samples - microarray and RNA-seq. This again indicates that these findings are reproducible across species, making these miRNAs strong potential bridging biomarkers of HIE. These miRNAs correlate positively and are predictive of $\mathrm{Lac} / \mathrm{Cr}$ and Lac/NAA MRS measurements, suggesting that, if validated in humans, they could potentially be used in place of MRS for more timely, cheaper, more accessible HIE diagnoses. The functional and cellular response to miR-181a inhibition outlined in the present manuscript is novel and provides a critically important starting point for further investigation into miRNAs as therapeutic targets for HIE. Whole blood was advantageous to use for this study due to its high miRNA concentrations and for ease of use in its least processed form, as opposed to serum and plasma, where processing of small samples can result in low miRNA yields [87, 88]. If such a sample can be utilised to identify a biomarker, it offers the possibility of using finger/toe prick diagnostics, which would be ideal for HIE diagnosis in the fast-paced NICU setting. Another substantial advantage to the present study is the examination of miRNAs at all levels - in silico, in vitro and in vivo.

While this study has advantages, we must also address limitations. RNA-Seq unfortunately did not detect any miRNA differences between mild HI and moderate-severe HI. This is a major limitation for the clinical relevance of this study, as detection of mild HIE is notoriously difficult and can result in poor outcomes [89]. Both the human and porcine cohort grouped neonates with moderate and severe HIE together, as numbers were insufficient to examine moderate and severe HI separately. In doing so, we therefore may be missing important differences between the two groups. Future clinical studies may wish to separate the groups to capture these differences. An important limitation of the current study is the small sample size in the mild HI subgroup, as the model is designed to produce moderate-severe HI. Therefore, findings must be interpreted with caution. Our current findings warrant further research using much larger sample sizes. We acknowledge that not all miRNAs identified using our human cohort showed a change in HI piglets. Many clinical studies of miRNA alterations in HIE find our miRNAs of interest to be downregulated $[5,56]$, while we and others have seen the opposite effect in animals [57]. It is unclear as to whether these are cross-species differences or whether it simply reflects the unpredictable nature and severity of $\mathrm{HI}$ in the clinical setting, as our porcine cohort received a uniform insult. miRNA alterations in both control and HI piglets show considerable variability over time. Results should be interpreted cautiously, and larger studies are required to increase confidence in the findings. It is difficult to determine whether our elevated miRNAs of interest promote neuroprotective effects or play a detrimental role in the HI brain, as miRNAs appear to exert individual synaptogenic functions [58]. Although whole blood samples are advantageous/easy to use, we must also consider it contains variable exosomal miRNA content [90]. Another limitation of this study is the use of U6 as a housekeeping gene. Although it is widely used in miRNA studies, expression is often variable and stability may be affected by species, experimental variables, pathological conditions [91] and number of freeze-thaw cycles. Stable housekeeping genes suitable for 
miRNA research are yet to be identified. Ultimately, miRNA research lacks suitable endogenous control genes, and there is an unmet need for these to allow more reliable, reproducible results in the field [92]. When interpreting the present in vitro findings, we must consider the effect of developmentally immature E14 neuronal cultures on the intervention, which may affect translatability. Neurogenesis in E14 rats is equivalent to that seen in a human at gestational week 5-6 [93]. The rat brain at either PND 7 or PND 12/13 would be more developmentally appropriate to use in future studies [94]. Longer time course experiments with larger sample numbers are required to identify whether we will observe a similar effect as is seen here. Finally, the present manuscript lacks investigation into functional effects of miR inhibition in hypoxic cells, as it was beyond the remit of the study.

In summary, this study provides novel insight into temporal profiles of circulating miRNAs after moderate-severe $\mathrm{HI}$ in a porcine model and begins to explore functional effects of miRNA inhibition in vitro. Further research into the roles of these miRNAs and their targets to confirm specific mechanisms of action in HI brain injury is required. miR-181a inhibition increases neurite growth in healthy cells, providing a starting point for further investigation into the therapeutic potential of miRNA inhibition in HIE. The rapid evolution of miRNA expression, coupled with positive correlations with $\mathrm{Lac} / \mathrm{Cr}$ ratios indicates towards their potential to be early bridging biomarkers of HI, particularly moderate-severe HI, detectable almost immediately after birth in neonates and piglet models, during the window in which time-sensitive decisions regarding therapeutic interventions are required.

\section{Materials and Methods}

\section{Microarray Study Population}

Differentially expressed HIE-associated miRNAs were identified from an exploratory human cohort using Agilent Human miRNA Microarray Version 3.0 (Agilent Technologies Inc., Agilent Laboratories, Santa Clara, CA). This contains probes for 866 human-derived miRNAs from the Sanger miRBase 12.0 release (http://www.mirbase.org). Microarray was performed in a previous study utilising a study population which was previously described in detail [5].

\section{RNA Sequencing}

\section{Study Population}

Umbilical cord blood samples (mixed venous and arterial) were collected from infants in both Cork University Maternity Hospital and Karolinska University Hospital. All relevant consent and ethical approval were obtained from both centres. These samples were obtained from infants recruited to the BiHiVE 1 and BiHiVE2 studies (NCT02019147). A total of 48 samples were sequenced: 19 female, 29 male. Of these, samples were collected from 35 infants in Cork (15 female, 20 male) and 13 in Karolinska (4 female, 9 male).

All cases were inborn and were recruited to the study if they met the following criteria: Gestational age of $\geq 36$ weeks accompanied by one or more of the following: $\mathrm{Cord} \mathrm{pH}<7.1$, 5-min Apgar score of $\leq 6$ or experienced intubation or resuscitation at birth. Case neonates were monitored throughout the perinatal period to ensure detection of potential HIE development. All HIE neonates received multichannel EEG at 24-h postpartum, and this was used to correlate with Sarnat HIE grade $[95,96]$. Infants who did not develop HIE were assigned to the perinatal asphyxia (PA) group. These infants has biochemical and clinical signs of $\mathrm{PA} ; \mathrm{pH}<7.1$ or Apgar score $<6$ at $5 \mathrm{~min}$, or the need for resuscitation and cardiorespiratory support at birth [97], but did not progress to HIE. Healthy controls were full term infants with uneventful births, normal Apgar score, normal neonatal exams and no record of admission to the neonatal intensive care unit. Both studies were approved respectively by the Clinical Ethics Committee of the Cork Teaching Hospitals and the Stockholm Ethical Review Board, and informed written consent was given by parents.

\section{Biofluid Collection}

Umbilical cord blood samples were collected directly after delivery in vacutainer tubes $(6 \mathrm{ml}$ collected $)$ and processed within $3 \mathrm{~h}$. Samples were biobanked at $-80^{\circ} \mathrm{C}$ until required for further analysis. Plasma was separated out from whole blood via centrifugation of the tubes at $2400 \times g$ for $10 \mathrm{~min}$ at $4{ }^{\circ} \mathrm{C}$. Supernatant was collected in RNAase-/DNAase-free tubes, taking care not to disturb the buffy coat which contains the white blood cells. Plasma was collected in $250 \mu$ RNAase free Eppendorf tubes and biobanked at $-80^{\circ} \mathrm{C}$ until required. Haemolysis was determined via spectrophotometric analysis with a Nanodrop 2000 spectrophotometer (Thermo Scientific, Wilmington, Delaware, USA). The absorbance levels at $414 \mathrm{~nm}$ was assessed and a cutoff level of $0.25 \mathrm{~nm}$ was utilised to distinguish non-haemolysed samples.

\section{Small RNA Library Preparation \& RNA-Seq}

A total of $200 \mu \mathrm{l}$ of the aforementioned non-haemolysed plasma samples was utilised for RNA isolation. The miRCURY RNA isolation kit (Exiqon) was used for this purpose, as per manufacturer's instructions. RNA samples were eluted to $25 \mu \mathrm{l}$. RNA ethanol precipitation was performed overnight, after which RNA was resuspended in $10 \mu$ RNAse-free water. The integrity and amount of RNA was determined using the small RNA detection kit (DNF-470) and a fragment analyser 
(Advanced Analytical Technologies). Control samples contained between 10.2 and $79.7 \%$ miRNA content, and miRNA concentrations ranged between 714.6 and $9594.7 \mathrm{pg} / \mu \mathrm{l}$. PA samples contained between 23.7 and 92.4\% miRNA content, and miRNA concentrations ranged between 5.3 and 53,104 pg/ $\mu$ l. Mild samples contained between 17.4 and $74.5 \%$ miRNA content, and miRNA concentrations ranged between 651.6 and $3960.8 \mathrm{pg} / \mu \mathrm{l}$. Moderate samples contained between 24.9 and $53.6 \%$ miRNA content, and miRNA concentrations ranged between 923.6 and $5551.9 \mathrm{pg} / \mu \mathrm{l}$. Severe samples contained between 26.9 and $81.4 \%$ miRNA content, and miRNA concentrations ranged between 2787.4 and $17,571.3 \mathrm{pg} / \mu \mathrm{l}$.

A total of $5 \mu$ l of RNA from each sample was used to create small RNA libraries using the Illumina TruSeq Small RNA library kit. These libraries were then size selected using Pippin Prep with 3\% agarose dye-free cassettes. Size selection was validated with a 2100 high sensitivity DNA bioanalyser chip (Agilet). Concentration levels of each library were determined using HS-dsDNA kit for Qubit, and these libraries were pooled. Pooled libraries were sequenced at TrinSeq (Institute for Molecular Medicine, St. James Hospital, Dublin), using an Illumina miSeq.

\section{Sequencing Data Processing and Differential miRNA Analysis}

Quality of reads was assessed using FastQC [98]. The files generated were uploaded to Chimira [28], which generated count-based miRNA expression data. Sequences were then adaptor trimmed and mapped out against miRbase v21 hairpin sequences, allowing 2 or fewer sequence mismatches. Further analysis was performed with R/Bioconductor (http://www.Rproject.org) [99, 100]. EdgeR [101] identified significantly differentially expressed miRNAs according to Law et al.'s protocol [102]. The trimmed mean of $M$ values (TMM) normalisation method was used for normalisation of miRNA expression count data. Raw counts were converted to CPM and $\log$-CPM values using the CPM function in edgeR, where logtransformations use a prior count of 0.25 to avoid taking the $\log$ of zero. A miRNA was considered to be expressed if their CPM value was above 1 , and not expressed otherwise. A CPM of 1 is equivalent to a log-CPM of 0 . A miRNA must be expressed in at least 30 of the 39 samples across the entire experiment in order to be included in subsequent analysis. Filtered data was then normalised using the TMM method [103].

Like other studies, hsa-miR-486-5p was the most highly expressed miRNA. It is one of the most abundant miRNAs found in plasma and makes up 13\% of all miRNAs [104, 105]. In the most extreme cases, it made up for $92 \%$ of the total number of counts (range $=8-92 \%$; average $=61 \%$ ). Therefore, we removed this miRNA from the analysis, recalculated the counts and removed nine samples with less than 2000 counts (IDs 193, 81, 830, 532, 93, 37, 110, 314 and 602). This included two samples where over $90 \%$ of the counts in the sample were hsa-miR-486-5p. A total of 39 samples remained: 7 control, 10 PA, 14 mild, 8 moderatesevere.

miRNAs of interest were selected based on the combined findings from both the microarray and RNA-seq, as well as previous findings from our group (Table 4). Significant miRNAs which were regulated in the same direction in both the microarray and the RNA-seq were selected for investigation in piglet whole blood taken over a 72-h time course, as well as CNS-enriched miR-27b, miR-128, miR-151a, miR181b, miR-374a and miR-376a as they are of interest within our research group and had shown significant alterations in either the microarray or RNA-seq.

\section{Piglet Model of HIE}

The neonatal animal model utilised in this study was the Large White piglet. Piglets were less than $24 \mathrm{~h}$ old with a mean(SD) age of 17.7(4.52)h for HI animals and 10.5(3.72)h for controls. Piglet weight ranged between 1.2 and $2.1 \mathrm{~kg}$, with HI animals weighing $1.56(0.26) \mathrm{kg}$, and controls weighing $1.52(0.18) \mathrm{kg}$. Age at postmortem was $54(25.5) \mathrm{h}$ for HI animals, and 75.69(3.59)h for controls. All animal work was performed under the supervision of Dr. Tracey Bjorkman, University of Queensland, Australia. Approval was obtained from the University of Queensland Animal Experimentation Ethics Committee and was performed in accordance with the National Health and Medicine Research Council Australia guidelines. Ten piglets received HI, while 10 were assigned to the sham experimental protocol. In the HI group, 7 were males and 3 were females. The control group consisted of 6

Table 4 Direction of regulation of miRNAs in both microarray (whole blood) and RNA-seq (plasma), along with references to involvement of each miRNA in disease/disordered states

\begin{tabular}{llll}
\hline miRNA & Microarray & RNA-seq & Literature \\
\hline miR-27b & Downregulated & Upregulated & {$[106]$} \\
miR-30b & Downregulated & Downregulated & {$[107,108]$} \\
miR-30c & Downregulated & Downregulated & {$[109-111]$} \\
miR-128 & Downregulated & Upregulated & {$[112-114]$} \\
miR-140 & Downregulated & Downregulated & {$[115-117]$} \\
miR-148a & Downregulated & Downregulated & {$[74,118,119]$} \\
miR-151a & - & Downregulated & {$[46,48,49]$} \\
miR-181a & Downregulated & Downregulated & {$[52,107,120]$} \\
miR-181b & - & Downregulated & {$[52,107,108,121]$} \\
miR-374a & Downregulated & - & {$[5,122,123]$} \\
miR-376a & Downregulated & - & {$[124]$} \\
\hline
\end{tabular}


males and 4 females. Three HI animals displayed clinical seizures, two of which also displaying seizure activity on EEG.

Anaesthesia was achieved initially via inhalation of isofluorane, the ear vein cannulated for maintenance of sedation was maintained using propofol $(10 \mathrm{mg} / \mathrm{ml})$ and Alfentanil $(50 \mu \mathrm{g} / \mathrm{ml} ; 0.5 \mathrm{mg} / 1 \mathrm{ml}$, Rapifen, VIC, Australia) as an analgesic. The aforementioned mixture was delivered at a rate of $10 \mathrm{mg} / \mathrm{kg} / \mathrm{h}$ until the point of intubation (size 3, cuffed endotracheal tube). At this point, delivery rate was increased to $20 \mathrm{mg} / \mathrm{kg} / \mathrm{h}$ for $15 \mathrm{~min}$, reduced thereafter to $15 \mathrm{mg} / \mathrm{kg} / \mathrm{h}$ for a further $15 \mathrm{~min}$ then maintained at a rate of $10 \mathrm{mg} / \mathrm{kg} / \mathrm{h}$ until termination of HI.

Ventilation was achieved using a neonatal ventilator (SLE2000, Surrey, UK), set initially at 30 breaths per minute, peak inspiratory pressure at $12 \mathrm{~cm} \mathrm{H}_{2} \mathrm{O}$, positive-end expiratory pressure at $5 \mathrm{~cm} \mathrm{H}_{2} \mathrm{O}, 0.75$-s inspiratory time and $21 \%$ inspired oxygen $\left(\mathrm{iO}_{2}\right)$. Adjustments were made throughout the duration of the experiment for maintenance of arterial oxygen saturation $>96 \%$ and a partial pressure of $\mathrm{CO}_{2}$ between 35 and $45 \mathrm{mmHg}$.

A catheter (3.5-French gauge single lumen) was inserted aseptically into the umbilical artery to approx. $12 \mathrm{~cm}$ to facilitate arterial blood sampling and monitoring of mean arterial blood pressure (MABP). Arterial blood samples were taken pre-insult, during $\mathrm{HI}$ at $0 \mathrm{~h}$ (immediately prior to resuscitation), and 1-h, 2-h, 4-h, 8-h, 12-h, 24-h, 48-h and 72-h postHI. Arterial blood gases were monitored (ABL800, Radiometer Pacific Pty. Ltd., Brisbane, Australia) throughout $\mathrm{HI}$, allowing titration of normal arterial $\mathrm{pO}_{2}, \mathrm{pCO}_{2}, \mathrm{pH}$ and ABE levels. Piglets received a $0.2 \mathrm{mg} / \mathrm{kg}$ single doses of iv cephalothin (DBL, $100 \mathrm{mg} / \mathrm{ml}$, VIC, Australia) and $0.25 \mathrm{mg} / \mathrm{kg}$ gentamicin (DBL, $10 \mathrm{mg} / \mathrm{ml}$, VIC, Australia) after umbilical catheterisation. Glucose infusion (10\%@3 ml/kg/ h) was maintained for the experiment duration.

Piglets were allowed to stabilise for 85 to $100 \mathrm{~min}$ from time of initial anaesthetic induction. Following this, $\mathrm{iO}_{2}$ was reduced from levels of $21 \%$ down to $4 \%$ for approx. $30 \mathrm{~min}$ to induce hypoxia. This was adjusted to achieve a moderatesevere injury. HI was titrated to individual physiological responses based on low amplitude EEG $(<5 \mu \mathrm{V}), \mathrm{pH}, \mathrm{MABP}$ and $\mathrm{ABE}$ levels. HI was terminated by resuscitation with $21 \%$ $\mathrm{iO}_{2}$. Arterial blood gases are recorded at 15, 30, 45 and $60 \mathrm{~min}$ after $\mathrm{HI}$ and every $4 \mathrm{~h}$ thereafter. Anaesthetic regimen was reduced to $4 \mathrm{mg} / \mathrm{kg}$ and adjusted where necessary (4$12 \mathrm{mg} / \mathrm{kg}$ ) until cessation of anaesthesia at 36-h post-HI. These parameters for induction of $\mathrm{HI}$ have been demonstrated previously and were shown to induce a moderate-severe HI accompanied by spontaneous seizures [15].

A rectal temperature of $38.5 \pm 0.2^{\circ} \mathrm{C}$ was maintained using an overhead radiant heater. Heart rate (HR), oxygen saturation $\left(\mathrm{SO}_{2}\right), \mathrm{MABP}$ and rectal temperature were recorded continuously (Marquette Tramscope 12C, Medical Systems, WI, USA). EEG recordings were taken with a cerebral function monitor (Unique CFM 6.0, Inspiration Healthcare, Leicester, UK) for experiment duration until anaesthesia was ceased (up to 36 -h post-HI in piglets surviving to $72 \mathrm{~h}$ ). Electrodes were located over frontal and parietal cortices, and a reference electrode was also placed at the neck fat pad. EEG recordings were also performed for approx. $1 \mathrm{~h}$ at both the 48 - $\mathrm{h}$ and 72-h timepoints post-HI.

In the HI group, final numbers of piglet blood samples were as follows: $n=10$ at $0 \mathrm{~h}, n=10$ at $1 \mathrm{~h}, n=10$ at $2 \mathrm{~h}$, $n=10$ at $8 \mathrm{~h}$ and $n=5$ at $72 \mathrm{~h}$. In the healthy control group, final $\mathrm{n}$ numbers were as follows: $n=9$ at $0 \mathrm{~h}, n=9$ at $1 \mathrm{~h}, n=$ 9 at $2 \mathrm{~h}, n=7$ at $8 \mathrm{~h}$ and $\mathrm{n}=9$ at $72 \mathrm{~h}$.

\section{SH-SY5Y and E14 Cell Culture and Transfection}

For imaging of BMP response to miR-181a inhibition, SHSY5Y and embryonic (E) day 14 cells from rat ventral mesencephalon (VM) were grown in vitro as previously described [125] with full ethical approval. SH-SY5Ys were cultured with the addition of $20 \mu \mathrm{M}$ retinoic acid to allow differentiation into a more neuronal phenotype [126], in a 24-well plate at 50,000 cells/well respectively over 3 separate passages. After $24 \mathrm{~h}$, cells were at $80 \%$ confluency and were transfected using the TransIT-X2 Dynamic Delivery System (Mirus Bio, Madison, WI, USA) as per manufacturer's instructions, GFP plasmid and $500 \mathrm{nM}$ mirVana miR-181a Inhibitor or Negative Control \#1 (Life Technologies, Grans Island, NY, USA).

\section{RNA Isolation}

Umbilical artery whole blood samples were collected using RNAprotect Animal Blood Tubes and biobanked at $-80{ }^{\circ} \mathrm{C}$. Total RNA including miRNA was extracted using the Qiagen RNeasy Protect Animal Blood kit (Qiagen, Hilden, Germany) as per manufacturer's instructions, with additional $80 \%$ ethanol and buffer RPE washes to increase the purity of the samples. Isolated RNA was stored at $-80{ }^{\circ} \mathrm{C}$ until required. Total RNA concentrations were quantified using a Nanodrop 8000 Spectrophotometer (Thermo Scientific).

\section{qRT-PCR}

Eleven miRNAs were selected for further investigation based on microarray and RNA-seq findings. Extracted RNA samples were diluted using nuclease free water to achieve a concentration of $20 \mathrm{ng} / \mu \mathrm{l}$ prior to cDNA synthesis. cDNA was synthesised using the miRCURY LNA Universal RT microRNA PCR kit (Exiqon, Vedbank, Denmark) as per manufacturer's instructions. qRT-PCR was performed on the StepOnePlus RT-PCR System (Applied Biosystems, CA, USA) using miRCURY LNA microRNA PCR ExiLENT SYBR Green master mix (Exiqon) as per the manufacturer's instructions for hsa-miR-27b-3p, hsa-miR-30b-5p, hsa-miR- 
30c-5p, hsa-miR-128-3p, dre-miR-140-3p, hsa-miR-148a-5p, hsa-miR-151a-5p, hsa-miR-181a-3p, ssc-miR-181b, hsamiR-374a-5p and hsa-miR-376a-3p. To achieve this, predesigned primers (Exiqon) were used. The sequences $\left(5^{\prime}-\right.$ $3^{\prime}$ ) of these are listed in Table 5. The sequences of all miRNAs, except miR-140, miR-181a and miR-376a have good sequence homology with human miRNA. A total of $4 \mu \mathrm{l}$ of diluted template cDNA was used per $10 \mu \mathrm{l}$ reaction. U6 SNRNA, a small nuclear RNA, was used as a reference gene as it is frequently utilised for studies of miRNA expression such as this [127, 128]. UniSP6 was spiked in during cDNA synthesis, acting as an internal control.

The expression of these miRNAs was examined in both HI and control samples at $0 \mathrm{~h}, 1 \mathrm{~h}, 2 \mathrm{~h}, 8 \mathrm{~h}$ and $72 \mathrm{~h}-$ immediately prior to resuscitation, during the primary injury phase and the secondary injury/reperfusion phase.

Each sample was run in triplicate, with cycling threshold (CT) values recorded for each. Levels of miRNA expression were calculated relative to the reference gene using the $2^{-} \Delta \Delta \mathrm{CT}$ method [129].

\section{Immunocytochemistry of SH-SY5Y Cells}

Cells were fixed in 4\% PFA (Sigma-Aldrich) for $25 \mathrm{~min}$ at RT before $3 \times$ washes with $0.02 \%$ Triton-X-100 (Sigma Aldrich) in $10 \mathrm{mM}$ PBS with $5 \mathrm{~min}$ between each wash. Following this, cells were blocked for $1 \mathrm{~h}$ at RT with $5 \%$ bovine serum albumin (BSA) in $10 \mathrm{mM}$ PBS. Primary antibodies for BMP2, BMPR2, BMPR1B and ASK1 (Abcam, Cambridge, UK) were used at a 1:200 dilution in 1\% BSA, added to wells and incubated at $4{ }^{\circ} \mathrm{C}$ overnight. Following this, cells were again washed $3 \times$ and incubated with appropriate AlexaFluor 594-conjugated secondary antibodies (ThermoFisher Scientific) at a 1:500 dilution in 1\% BSA and incubated in the dark at RT for $2 \mathrm{~h}$. Cells were washed for a final $3 \times$ times before being imaged on an Olympus IX71 using a $\times 20$ lens.
Five fields were imaged per group (10 fields/N). Cells cotransfected with GFP and miRNA inhibitor appear green in Fig. 7.

\section{Analysis of Cellular Morphology and BMP Activity}

To assess the cellular morphology of miR-181a inhibitortransfected cells, neurite length was measured for five random neurites per field for GFP co-transfected cells. Somal area and fluorescence were also measured for five random neurons per field for GFP co-transfected cells. All experiments were repeated three times, with all analysis performed using ImageJ.

\section{Histopathology of Piglet Brain}

Piglets were euthanised with an intraperitoneal injection of sodium pentabarbitone $(325 \mathrm{mg} / \mathrm{kg})$ and the brain was intracardially flushed with saline. Brains were then removed, coronally sliced $(3 \mathrm{~mm})$ and hemisected. The right hemisphere was fixed in $4 \%$ paraformaldehyde, $0.1 \mathrm{M}$ phosphate buffered saline (PBS pH 7.4), with agitation overnight at room temperature. Brains were stored in $0.1 \%$ paraformaldehyde $/ 0.1 \mathrm{M}$ PBS until paraffin embedding. The left hemisphere was dissected into eight regions of interest and snap frozen in liquid nitrogen before storage at $-80{ }^{\circ} \mathrm{C}$.

To assess histopathological injury, $4-\mu \mathrm{m}$-thick paraffinembedded sections were dewaxed, rehydrated and stained with haematoxylin and eosin (H\&E) using an automated staining system (Leica ST5010 Autostainer XL, Leica Biosystems, North Ryde, NSW, Australia) and imaged using an Olympus BX41 light microscope with DP70 camera and $\times 20$ and $\times 40$ objectives. Two slides from each region including frontal and temporal cortices, basal ganglia, hippocampus, thalamus, cerebellum and brainstem were scored by a blinded observer. Histological injury (individual, laminar or confluent infarct)
Table 5 Sequence (5'-3') comparisons between hsa and ssc miRNAs. All have sequence homology (shown in green) apart from miR-140, miR-181a and miR-376a. miR-181b has good sequence homology, apart from an extra U base. Nonhomologous bases are shown in red

\begin{tabular}{lll}
\hline miRNA & Human (Hsa) & Pig (Ssc) \\
\hline miR-27b-3p & UUCACAGUGGCUAAGUUCUGC & UUCACAGUGGCUAAGUUCUGC \\
miR-30b-5p & UGUAAACAUCCUACACUCAGCU & UGUAAACAUCCUACACUCAGCU \\
miR-30c-5p & UGUAAACAUCCUACACUCUCAGC & UGUAAACAUCCUACACUCUCAGC \\
miR-128-3p & UCACAGUGAACCGGUCUCUUU & UCACAGUGAACCGGUCUCUUU \\
miR-140-3p & UACCACAGGGUAGAACCACGG & UACCACAGGGUAGAACCACGGAC \\
miR-148a-5p & AAAGUUCUGAGACACUCCGACU & AAAGUUCUGAGACACUCCGACU \\
miR-151a-5p & UCGAGGAGCUCACAGUCUAGU & UCGAGGAGCUCACAGUCUAGU \\
miR-181a-3p & ACCAUCGACCGUUGAUUGUACC & AACAUUCAACGCUGUCGGUGAGUU \\
miR-181b & AACA & AACA \\
& UUCAUUGCUGUCGGUGGGU & UUCAUUGCUGUCGGUGGGUU \\
miR-374a-5p & UUAUAAUACAACCUGAUAAGUG & UUAUAAUACAACCUGAUAAGUG \\
miR-376a-3p & AUCAUAGAGGAAAAUCCACGU & GUAGAUUCUCCUUCUAUGAGUAC \\
\hline
\end{tabular}


and size of affected area $(<20 \%, 20-50 \%$ and $>50 \%)$ were determined as previously described [15].

Each brain region was assessed and scored between 0 and 9. A score of 0 inferring no injury was detected, 1-3 was classed as mild, 4-6 was moderate and 7-9 being severe injury (hippocampus scored only to a maximum of 6). Brain region scored was summated for each animal to reach a total histopathology score for statistical analysis. A total score between 0 and 15 was classed as mild brain injury, 16-30 was moderate injury and $31-45$ was severe injury.

\section{Magnetic Resonance Imaging}

MRS was performed on a 7-T Bruker ClinScan PET-MR scanner. A single spectrum was obtained in the frontoparietal area of the brain from a $15-\mathrm{mm}^{3}$ voxel utilising point resolved spectroscopy with the following parameters applied: $\mathrm{TR}=1500 \mathrm{~ms}, \mathrm{TE}=136 \mathrm{~ms}$ and 192 averages. Spectra were analysed with jMURI using the AMARES method and phased manually [130]. Apodisation (10 Hz) was applied to NAA, Cr and Cho peaks with Lorentzian peaks prior to quantification, while Lac was represented by a broad inverted peak. Lac was therefore quantified $180^{\circ}$ out of phase using the Gaussian peak. Ratios were determined for Lac/Cr, Lac/NAA, Lac/ Cho, Lac/Glx, Glx/NAA, Cho/Cr, NAA/Cho and NAA/Cr.

\section{In Silico Analysis of miRNA Target Genes}

Human miRNAs were searched on the miRDB database [131] which allowed retrieval of a list of predicted target genes. Each gene featured in the list was assigned a score. Scores $\geq$ 60 were deemed reliable, and those below were excluded due to uncertainty of validity, as per the miRDB guidelines [132, 133]. The lists of reliable target genes were inserted into http:// bioinformatics.psb.ugent.be/webtools/Venn/ which created tables of the genes which were targeted by a single miRNA along with common miRNA targets. These interactions were visualised as a chord diagram utilising the 'circilize' package in R 3.5.2 [134].

The previously generated lists of target gene symbols were then inserted into EnrichR [135] which allowed examination of the most significant KEGG pathways and gene ontologies (biological and molecular) associated with each list sorted by $p$ value. These lists were then cross-referenced to determine whether any miRNAs shared common pathways and common targets within these pathways.

\section{Statistical Analysis}

Differential expression analysis (Table 1) was performed using the voom and limma edgeR packages $[103,136$, 137]. Following on from this, empirical Bayesian moderation was applied by borrowing information across all
miRNAs to obtain more precise estimates of variability. Significance in miRNAs was defined using an adjusted $p$ value, with a 5\% cutoff [138]. Three contrasts were performed using the differentially expressed miRNAs identified in the RNA-seq data: moderate-severe vs control, moderate-severe vs mild and moderate-severe vs perinatal asphyxia.

All statistical analyses for physiological data, immunopathology and immunocytochemistry scores, MRS data and qRT-PCR findings were performed using IBM SPSS Statistics 24 (SPSS Inc., Chicago, Illinois, USA) and GraphPad Prism 7 (Graphpad Software Inc., San Diego, CA, USA). All data were analysed on a case versus control basis using two-way ANOVA with post hoc Fishers LSD test, independent samples $t$ tests or Mann-Whitney $U$ tests depending on distribution of the datasets. Statistical significance (2tailed) was set at $p \leq 0.05$. All $2 \Delta \Delta \mathrm{CT}$ qRT-PCR data was $\log _{10}$ transformed prior to analysis.

Spearman's correlations were performed for MRS ratios, scaled histology scores and miRNA expression absolute values at $1 \mathrm{~h}$. All values were reported as correlation coefficients $(r \square)$, with $p \leq 0.05$, and when corrected for multiple comparisons $p=0.0033$ with a Bonferroni correction applied. Linear regressions were performed for MRS ratios, scaled histology scores and miRNA expression values at $1 \mathrm{~h}$. All values were reported as $\left(R^{2}\right)$, with $p<0.05$.

Authors' Contributions S Casey wrote the manuscript, designed and performed all experiments on porcine whole blood and in vitro SH-SY5Y samples, in silico analysis and performed data analysis. K Goasdoue, SM Miller and ST Bjorkmann performed in vivo work, provided the blood samples, acquired representative images of stained tissue and commented on the manuscript at all stages. GP Brennan, C Mooney and DC Henshall performed and oversaw the RNA-seq and commented on the manuscript at all stages. G Cowin performed MRS. AG O'Mahony performed all E14 in vitro experiments, and participated in SH-SY5Y experiments, data analysis and commented on the manuscript. C Burke performed histology. B Hallberg and GB Boylan were involved in the development of the precursor study to this-BiHIVE. AM Sullivan was involved in supervision of E14 and SH-SY5Y experiments and data analysis. GW O'Keeffe was involved in study design, provided support and supervision for experimental and statistical issues and commented on the manuscript at all stages. DM Murray was involved in study design and supervision at all stages and commented on the manuscript at all stages.

Funding Information SC was supported by Irish Research Council GOIPG/2017/1350. DCH was supported by a research grant from Science Foundation Ireland (SFI) under grant number 16/RC/3948, cofunded under the European Regional Development Fund and by FutureNeuro industry partners and SFI by grants SFI/13/IA/1891 and SFI/14/ADV/RC2721. STB was supported by NHMRC Project Grant \#1084913 and Lions Medical Research Foundation (LMRF) Senior Fellowship. KG was supported by an Australian Research Training Program (RTP) Scholarship. The authors acknowledge the facilities and scientific and technical assistance of the National Imaging Facility, a National Collaborative Research Infrastructure Strategy (NCRIS) capability, at the Centre for Advanced Imaging, University of Queensland, Australia. 
Data Availability All data generated throughout the study which support the findings outlined in this manuscript are available from the corresponding author upon reasonable request.

\section{Compliance with Ethical Standards}

Conflict of Interest The authors declare that they have no conflict of interest.

Ethics Approval The BiHIVE $1 \& 2$ studies were approved respectively by the Clinical Ethics Committee of the Cork Teaching Hospitals and the Stockholm Ethical Review Board, and informed written consent was given by parents.

All animal work was performed under the supervision of Dr. Tracey Bjorkman, University of Queensland, Australia. Approval was obtained from the University of Queensland Animal Experimentation Ethics Committee and was performed in accordance with National Health and Medicine Research Council Australia guidelines.

\section{References}

1. Douglas-Escobar M, Weiss MD (2015) Hypoxic-ischemic encephalopathy: a review for the clinician. JAMA Pediatr 169(4): 397-403. https://doi.org/10.1001/jamapediatrics.2014.3269

2. Kurinczuk JJ, White-Koning M, Badawi N (2010) Epidemiology of neonatal encephalopathy and hypoxic-ischaemic encephalopathy. Early Hum Dev 86(6):329-338. https://doi.org/10.1016/j. earlhumdev.2010.05.010

3. Allen KA, Brandon DH (2011) Hypoxic ischemic encephalopathy: pathophysiology and experimental treatments. Newborn Infant Nurs Rev 11(3):125-133. https://doi.org/10.1053/j.nainr. 2011.07.004

4. DuPont TL, Chalak LF, Morriss MC, Burchfield PJ, Christie L, Sánchez PJ (2013) Short-term outcomes of newborns with perinatal acidemia who are not eligible for systemic hypothermia therapy. J Pediatr 162(1):35-41. https://doi.org/10.1016/j.jpeds.2012. 06.042

5. Looney AM, Walsh BH, Moloney G, Grenham S, Fagan A, O'Keeffe GW, Clarke G, Cryan JF et al (2015) Downregulation of umbilical cord blood levels of miR-374a in neonatal hypoxic ischemic encephalopathy. J Pediatr 167(2):269-273.e262. https:// doi.org/10.1016/j.jpeds.2015.04.060

6. McGuire W (2007) Perinatal asphyxia. BMJ Clin Evid 2007:0320

7. Vannucci RC, Perlman JM (1997) Interventions for perinatal hypoxic-ischemic encephalopathy. Pediatrics 100(6):1004-1014

8. Davidson JO, Wassink G, van den Heuij LG, Bennet L, Gunn AJ (2015) Therapeutic hypothermia for neonatal hypoxic-ischemic encephalopathy - where to from here? Front Neurol 6:198. https://doi.org/10.3389/fneur.2015.00198

9. Gunn AJ, Thoresen M (2006) Hypothermic neuroprotection. NeuroRX 3(2):154-169. https://doi.org/10.1016/j.nurx.2006.01. 007

10. Wassink G, Gunn ER, Drury PP, Bennet L, Gunn AJ (2014) The mechanisms and treatment of asphyxial encephalopathy. Front Neurosci 8:40. https://doi.org/10.3389/fnins.2014.00040

11. Khurshid F, Lee K-S, McNamara PJ, Whyte H, Mak W (2011) Lessons learned during implementation of therapeutic hypothermia for neonatal hypoxic ischemic encephalopathy in a regional transport program in Ontario. Paediatr Child Health 16(3):153156

12. Huang L, Zhao F, Qu Y, Zhang L, Wang Y, Mu D (2017) Animal models of hypoxic-ischemic encephalopathy: optimal choices for the best outcomes. Rev Neurosci 28(1):31-43. https://doi.org/10. 1515/revneuro-2016-0022

13. Volpe JJ (2012) Neonatal encephalopathy: an inadequate term for hypoxic-ischemic encephalopathy. Ann Neurol 72(2):156-166. https://doi.org/10.1002/ana.23647

14. Bjorkman ST, Miller SM, Rose SE, Burke C, Colditz PB (2010) Seizures are associated with brain injury severity in a neonatal model of hypoxia-ischemia. Neuroscience 166(1):157-167. https://doi.org/10.1016/j.neuroscience.2009.11.067

15. Björkman ST, Foster KA, O’Driscoll SM, Healy GN, Lingwood BE, Burke C, Colditz PB (2006) Hypoxic/ischemic models in newborn piglet: comparison of constant $\mathrm{FiO} 2$ versus variable FiO2 delivery. Brain Res 1100(1):110-117. https://doi.org/10. 1016/j.brainres.2006.04.119

16. Duhaime A-C, Margulies SS, Durham SR, O'Rourke MM, Golden JA, Marwaha S, Raghupathi R (2000) Maturationdependent response of the piglet brain to scaled cortical impact. J Neurosurg 93(3):455-462

17. Bartel DP (2004) MicroRNAs: genomics, biogenesis, mechanism, and function. Cell 116(2):281-297. https://doi.org/10.1016/ S0092-8674(04)00045-5

18. Qiu J, X-y Z, X-g Z, Cheng R, H-y L, Li Y (2013) Neuroprotective effects of microRNA-210 on hypoxic-ischemic encephalopathy. Biomed Res Int 2013:5-5. https://doi.org/10. $1155 / 2013 / 350419$

19. Ambros V (2001) microRNAs: tiny regulators with great potential. Cell 107(7):823-826. https://doi.org/10.1016/S00928674(01)00616-X

20. Xu P, Vernooy SY, Guo M, Hay BA (2003) The drosophila MicroRNA Mir-14 suppresses cell death and is required for normal fat metabolism. Curr Biol 13(9):790-795. https://doi.org/10. 1016/S0960-9822(03)00250-1

21. Kulshreshtha R, Ferracin M, Wojcik SE, Garzon R, Alder H, Agosto-Perez FJ, Davuluri R, Liu C-G et al (2007) A microRNA signature of hypoxia. Mol Cell Biol 27(5):18591867. https://doi.org/10.1128/MCB.01395-06

22. Moldovan L, Batte K, Wang Y, Wisler J, Piper M (2013) Analyzing the circulating microRNAs in exosomes/extracellular vesicles from serum or plasma by qRT-PCR. Methods Mol Biol (Clifton, NJ) 1024:129-145. https://doi.org/10.1007/978-162703-453-1_10

23. Matsumoto J, Stewart T, Banks WA, Zhang J (2017) The transport mechanism of extracellular vesicles at the blood-brain barrier. Curr Pharm Des 23(40):6206-6214. https://doi.org/10.2174/ 1381612823666170913164738

24. Lv H, Wang Q, Wu S, Yang L, Ren P, Yang Y, Gao J, Li L (2015) Neonatal hypoxic ischemic encephalopathy-related biomarkers in serum and cerebrospinal fluid. Clin Chim Acta 450:282-297. https://doi.org/10.1016/j.cca.2015.08.021

25. Ma Q, Dasgupta C, Li Y, Bajwa NM, Xiong F, Harding B, Hartman R, Zhang L (2016) Inhibition of microRNA-210 provides neuroprotection in hypoxic-ischemic brain injury in neonatal rats. Neurobiol Dis 89:202-212. https://doi.org/10.1016/j.nbd. 2016.02.011

26. Dharap A, Bowen K, Place R, Li L-C, Vemuganti R (2009) Transient focal ischemia induces extensive temporal changes in rat cerebral microRNAome. J Cereb Blood Flow Metab 29(4): 675-687. https://doi.org/10.1038/jcbfm.2008.157

27. Ziu M, Fletcher L, Rana S, Jimenez DF, Digicaylioglu M (2011) Temporal differences in microRNA expression patterns in astrocytes and neurons after ischemic injury. PLoS One 6(2):e14724. https://doi.org/10.1371/journal.pone.0014724

28. Vitsios DM, Enright AJ (2015) Chimira: analysis of small RNA sequencing data and microRNA modifications. Bioinformatics 31(20):3365-3367 
29. Zerbino DR, Achuthan P, Akanni W, Amode MR, Barrell D, Bhai J, Billis K, Cummins C et al (2018) Ensembl 2018. Nucleic Acids Res 46(D1):D754-D761. https://doi.org/10.1093/nar/gkx1098

30. Goulding SR, Sullivan AM, O'Keeffe GW, Collins LM (2019) Gene co-expression analysis of the human substantia nigra identifies BMP2 as a neurotrophic factor that can promote neurite growth in cells overexpressing wild-type or A53T $\alpha$-synuclein. Parkinsonism Relat Disord 64:194-201. https://doi.org/10.1016/ j.parkreldis.2019.04.008

31. Knight PG, Glister C (2006) TGF- $\beta$ superfamily members and ovarian follicle development. Reproduction 132(2):191-206

32. Li B, Dasgupta C, Huang L, Meng X, Zhang L (2019) MiRNA210 induces microglial activation and regulates microgliamediated neuroinflammation in neonatal hypoxic-ischemic encephalopathy. Cell Mol Immunol. https://doi.org/10.1038/ s41423-019-0257-6

33. Chen Z, Hu Y, Lu R, Ge M, Zhang L (2020) MicroRNA-374a-5p inhibits neuroinflammation in neonatal hypoxic-ischemic encephalopathy via regulating NLRP3 inflammasome targeted Smad6. Life Sci 252:117664. https://doi.org/10.1016/j.lfs.2020.117664

34. Stenvang J, Petri A, Lindow M, Obad S, Kauppinen S (2012) Inhibition of microRNA function by antimiR oligonucleotides. Silence 3(1):1. https://doi.org/10.1186/1758-907X-3-1

35. Nallamshetty S, Chan SY, Loscalzo J (2013) Hypoxia: a master regulator of microRNA biogenesis and activity. Free Radic Biol Med 64:20-30. https://doi.org/10.1016/j.freeradbiomed.2013.05. 022

36. Zhao M, Zhu P, Fujino M, Zhuang J, Guo H, Sheikh I, Zhao L, Li $\mathrm{X}-\mathrm{K}$ (2016) Oxidative stress in hypoxic-ischemic encephalopathy: molecular mechanisms and therapeutic strategies. Int J Mol Sci 17(12):2078. https://doi.org/10.3390/ijms17122078

37. Li M, Fu W, Wo L, Shu X, Liu F, Li C (2013) miR-128 and its target genes in tumorigenesis and metastasis. Exp Cell Res 319(20):3059-3064. https://doi.org/10.1016/j.yexcr.2013.07.031

38. Adlakha YK, Saini N (2013) miR-128 exerts pro-apoptotic effect in a p53 transcription-dependent and -independent manner via PUMA-Bak axis. Cell Death Dis 4:e542. https://doi.org/10.1038/ cddis.2013.46 https://www.nature.com/articles/cddis201346\# supplementary-information

39. Evangelisti C, Florian MC, Massimi I, Dominici C, Giannini G, Galardi S, Buè MC, Massalini S et al (2009) MiR-128 up-regulation inhibits Reelin and DCX expression and reduces neuroblastoma cell motility and invasiveness. FASEB J 23(12):4276-4287. https://doi.org/10.1096/fj.09-134965

40. Khan AP, Poisson LM, Bhat VB, Fermin D, Zhao R, KalyanaSundaram S, Michailidis G, Nesvizhskii AI et al (2010) Quantitative proteomic profiling of prostate cancer reveals a role for miR-128 in prostate cancer. Mol Cell Proteomics 9(2):298 312

41. Zheng D, Haddadin S, Wang Y, Gu L-Q, Perry MC, Freter CE, Wang MX (2011) Plasma microRNAs as novel biomarkers for early detection of lung cancer. Int J Clin Exp Pathol 4(6):575-586

42. Mi S, Lu J, Sun M, Li Z, Zhang H, Neilly MB, Wang Y, Qian Z et al (2007) MicroRNA expression signatures accurately discriminate acute lymphoblastic leukemia from acute myeloid leukemia. Proc Natl Acad Sci 104(50):19971-19976

43. Ding G-C, Chen M, Wang Y-X, Rui C, Xu W, Ding H-J, Shi Z-H (2016) MicroRNA-128a-induced apoptosis in HTR-8/SVneo trophoblast cells contributes to pre-eclampsia. Biomed Pharmacother 81:63-70. https://doi.org/10.1016/j.biopha.2016.03.040

44. De Santi C, Melaiu O, Bonotti A, Cascione L, Di Leva G, Foddis R, Cristaudo A, Lucchi M et al (2017) Deregulation of miRNAs in malignant pleural mesothelioma is associated with prognosis and suggests an alteration of cell metabolism. Sci Rep 7(1):3140. https://doi.org/10.1038/s41598-017-02694-0
45. Hu B, Tang WG, Fan J, Xu Y, Sun HX (2018) Differentially expressed miRNAs in hepatocellular carcinoma cells under hypoxic conditions are associated with transcription and phosphorylation. Oncol Lett 15(1):467-474

46. Chiyomaru T, Yamamura S, Zaman MS, Majid S, Deng G, Shahryari V, Saini S, Hirata H et al (2012) Genistein suppresses prostate cancer growth through inhibition of oncogenic MicroRNA-151. PLoS One 7(8):e43812. https://doi.org/10. 1371/journal.pone.0043812

47. Zhou R, Wang R, Qin Y, Ji J, Xu M, Wu W, Chen M, Wu D et al (2015) Mitochondria-related miR-151a-5p reduces cellular ATP production by targeting CYTB in asthenozoospermia. Sci Rep 5: 17743. https://doi.org/10.1038/srep17743 https://www.nature. com/articles/srep17743\#supplementary-information

48. Vasu MM, Anitha A, Thanseem I, Suzuki K, Yamada K, Takahashi T, Wakuda T, Iwata K et al (2014) Serum microRNA profiles in children with autism. Mol Autism 5(1):40

49. Gupta S, Verma S, Mantri S, Berman NE, Sandhir R (2015) Targeting microRNAs in prevention and treatment of neurodegenerative disorders. Drug Dev Res 76(7):397-418

50. Looney AM, O'Sullivan MP, Ahearne CE, Finder M, FelderhoffMueser U, Boylan GB, Hallberg B, Murray DM (2018) Altered expression of umbilical cord blood levels of miR-181b and its downstream target mUCH-L1 in infants with moderate and severe neonatal hypoxic-ischaemic encephalopathy. Mol Neurobiol 56: 3657-3663. https://doi.org/10.1007/s12035-018-1321-4

51. O'Sullivan M, Looney A, Moloney GM et al (2018) Validation of altered umbilical cord blood microrna expression in neonatal hypoxic-ischemic encephalopathy. JAMA Neurol 76:333-341. https://doi.org/10.1001/jamaneurol.2018.4182

52. Shi L, Cheng Z, Zhang J, Li R, Zhao P, Fu Z, You Y (2008) hsamir-181a and hsa-mir-181b function as tumor suppressors in human glioma cells. Brain Res 1236:185-193. https://doi.org/10. 1016/j.brainres.2008.07.085

53. Peng Z, Li J, Li Y, Yang X, Feng S, Han S, Li J (2013) Downregulation of miR-181b in mouse brain following ischemic stroke induces neuroprotection against ischemic injury through targeting heat shock protein A5 and ubiquitin carboxyl-terminal hydrolase isozyme L1. J Neurosci Res 91(10):1349-1362

54. Zhang H, Zhong K, Lu M, Mei Y, Tan E, Sun X, Tan W (2018) Neuroprotective effects of isosteviol sodium through increasing CYLD by the downregulation of miRNA-181b. Brain Res Bull 140:392-401. https://doi.org/10.1016/j.brainresbull.2018.05.015

55. Li D, Jian W, Wei C, Song H, Gu Y, Luo Y, Fang L (2014) Downregulation of miR-181b promotes apoptosis by targeting CYLD in thyroid papillary cancer. Int J Clin Exp Pathol 7(11):7672-7680

56. Wang Z, Liu Y, Shao M, Wang D, Zhang Y (2018) Combined prediction of miR-210 and miR-374a for severity and prognosis of hypoxic-ischemic encephalopathy. Brain Behav 8(1):e00835. https://doi.org/10.1002/brb3.835

57. Garberg HT, Huun MU, Baumbusch LO, Åsegg-Atneosen M, Solberg R, Saugstad OD (2017) Temporal profile of circulating microRNAs after global hypoxia-ischemia in newborn piglets. Neonatology 111(2):133-139

58. Ponnusamy V, Yip PK (2019) The role of microRNAs in newborn brain development and hypoxic ischaemic encephalopathy. Neuropharmacology 149:55-65. https://doi.org/10.1016/j. neuropharm.2018.11.041

59. Gomes JR, Nogueira RS, Vieira M, Santos SD, Ferraz-Nogueira JP, Relvas JB, Saraiva MJ (2016) Transthyretin provides trophic support via megalin by promoting neurite outgrowth and neuroprotection in cerebral ischemia. Cell Death Differ 23(11):17491764. https://doi.org/10.1038/cdd.2016.64

60. Zhao W, Yan J, Gao L, Zhao J, Zhao C, Gao C, Luo X, Zhu X (2017) $\mathrm{Cdk} 5$ is required for the neuroprotective effect of transforming growth factor- $\beta 1$ against cerebral ischemia- 
reperfusion. Biochem Biophys Res Commun 485(4):775-781. https://doi.org/10.1016/j.bbrc.2017.02.130

61. O'Driscoll CM, Gorman AM (2005) Hypoxia induces neurite outgrowth in PC12 cells that is mediated through adenosine A2A receptors. Neuroscience 131(2):321-329. https://doi.org/ 10.1016/j.neuroscience.2004.11.015

62. Li Q-J, Chau J, Ebert PJR, Sylvester G, Min H, Liu G, Braich R, Manoharan M et al (2007) miR-181a is an intrinsic modulator of T cell sensitivity and selection. Cell 129(1):147-161. https://doi.org/ 10.1016/j.cell.2007.03.008

63. Ouyang Y-B, Stary CM, Yang G-Y, Giffard R (2013) microRNAs: innovative targets for cerebral ischemia and stroke. Curr Drug Targets 14(1):90-101

64. Kong H, Omran A, Ashhab MU, Gan N, Peng J, He F, Wu L, Deng X et al (2014) Changes in microglial inflammation-related and brain-enriched microRNAs expressions in response to in vitro oxygen-glucose deprivation. Neurochem Res 39(2):233-243. https://doi.org/10.1007/s11064-013-1209-7

65. Choi K-Y, Kim H-J, Lee M-H, Kwon T-G, Nah H-D, Furuichi T, Komori T, Nam S-H et al (2005) Runx2 regulates FGF2-induced Bmp2 expression during cranial bone development. Dev Dyn 233(1):115-121. https://doi.org/10.1002/dvdy.20323

66. Wu M, Chen G, Li Y-P (2016) TGF- $\beta$ and BMP signaling in osteoblast, skeletal development, and bone formation, homeostasis and disease. Bone Res 4:16009. https://doi.org/10.1038/ boneres.2016.9

67. Chang H, Brown CW, Matzuk MM (2002) Genetic analysis of the mammalian transforming growth factor- $\beta$ superfamily. Endocr Rev 23(6):787-823. https://doi.org/10.1210/er.2002-0003

68. Wang RN, Green J, Wang Z, Deng Y, Qiao M, Peabody M, Zhang Q, Ye J et al (2014) Bone morphogenetic protein (BMP) signaling in development and human diseases. Genes Dis 1(1):87-105. https://doi.org/10.1016/j.gendis.2014.07.005

69. Sun E, Shi Y (2015) MicroRNAs: Small molecules with big roles in neurodevelopment and diseases. Exp Neurol 268:46-53. https://doi.org/10.1016/j.expneurol.2014.08.005

70. Kim M-J, Park SY, Chang HR, Jung EY, Munkhjargal A, Lim JS, Lee M-S, Kim Y (2017) Clinical significance linked to functional defects in bone morphogenetic protein type 2 receptor, BMPR2. BMB Rep 50(6):308-317. https://doi.org/10.5483/ bmbrep.2017.50.6.059

71. Beppu H, Kawabata M, Hamamoto T, Chytil A, Minowa O, Noda T, Miyazono K (2000) BMP type II receptor is required for gastrulation and early development of mouse embryos. Dev Biol 221(1):249-258. https://doi.org/10.1006/dbio.2000.9670

72. Samanta J, Alden T, Gobeske K, Kan L, Kessler John A (2010) Noggin protects against ischemic brain injury in rodents. Stroke 41(2):357-362. https://doi.org/10.1161/STROKEAHA.109. 565523

73. Liu Z, Shen J, Pu K, Katus HA, Plöger F, Tiefenbacher CP, Chen X, Braun T (2009) GDF5 and BMP2 inhibit apoptosis via activation of BMPR2 and subsequent stabilization of XIAP. Biochim Biophysi Acta (BBA) Mol Cell Res 1793(12):1819-1827. https:// doi.org/10.1016/j.bbamcr.2009.09.012

74. Zhang H, Li Y, Huang Q, Ren X, Hu H, Sheng H, Lai M (2011) MiR-148a promotes apoptosis by targeting Bcl-2 in colorectal cancer. Cell Death Differ 18:1702-1710. https://doi.org/10.1038/ cdd.2011.28

75. Yang LJ, Ma DQ, Cui H (2014) Proteomic analysis of immature rat pups brain in response to hypoxia and ischemia challenge. Int $\mathrm{J}$ Clin Exp Pathol 7(8):4645-4660

76. DiLella AG (1991) Chromosomal assignment of the human immunophilin FKBP-12 gene. Biochem Biophys Res Commun 179(3):1427-1433. https://doi.org/10.1016/0006-291X(91) 91732-R
77. Kwapiszewska G, Wilhelm J, Wolff S, Laumanns I, Koenig IR, Ziegler A, Seeger W, Bohle RM et al (2005) Expression profiling of laser-microdissected intrapulmonary arteries in hypoxiainduced pulmonary hypertension. Respir Res 6:109. https://doi. org/10.1186/1465-9921-6-109

78. Chen A, Xiong L-J, Tong Y, Mao M (2013) The neuroprotective roles of BDNF in hypoxic ischemic brain injury. Biomed Rep 1(2):167-176

79. Dhobale $\mathrm{M}$ (2014) Neurotrophins: role in adverse pregnancy outcome. Int J Dev Neurosci 37:8-14. https://doi.org/10.1016/j. ijdevneu.2014.06.005

80. Dobbing J, Sands J (1973) Quantitative growth and development of human brain. Arch Dis Child 48(10):757-767

81. Vannucci RC (2000) Hypoxic-ischemic encephalopathy. Am J Perinatol 17(3):113-120. https://doi.org/10.1055/s-2000-9293

82. Lai MC, Yang SN (2011) Perinatal hypoxic-ischemic encephalopathy. J Biomed Biotechnol 2011:1-6. https://doi.org/10.1155/ 2011/609813

83. Thayyil S, Chandrasekaran M, Taylor A, Bainbridge A, Cady EB, Chong WKK, Murad S, Omar RZ et al (2010) Cerebral magnetic resonance biomarkers in neonatal encephalopathy: a meta-analysis. Pediatrics 125:e382-e395

84. Robertson NJ, Thayyil S, Cady EB, Raivich G (2014) Magnetic resonance spectroscopy biomarkers in term perinatal asphyxial encephalopathy: from neuropathological correlates to future clinical applications. Curr Pediatr Rev 10(1):37-47

85. Hanrahan JD, Cox IJ, Azzopardi D, Cowan FM, Sargentoni J, Bell JD, Bryant DJ, Edwards AD (1999) Relation between proton magnetic resonance spectroscopy within 18 hours of birth asphyxia and neurodevelopment at 1 year of age. Dev Med Child Neurol 41(2):76-82. https://doi.org/10.1017/S0012162299000171

86. Ancora G, Soffritti S, Lodi R, Tonon C, Grandi S, Locatelli C, Nardi L, Bisacchi $\mathrm{N}$ et al (2010) A combined a-EEG and MR spectroscopy study in term newborns with hypoxic-ischemic encephalopathy. Brain and Development 32(10):835-842. https:// doi.org/10.1016/j.braindev.2009.11.008

87. Schultz NA, Dehlendorff C, Jensen BV, Bjerregaard JK, Nielsen KR, Bojesen SE, Calatayud D, Nielsen SE et al (2014) Microrna biomarkers in whole blood for detection of pancreatic cancer. JAMA 311(4):392-404. https://doi.org/10.1001/jama.2013. 284664

88. Patnaik SK, Yendamuri S, Kannisto E, Kucharczuk JC, Singhal S, Vachani A (2012) MicroRNA expression profiles of whole blood in lung adenocarcinoma. PLoS One 7(9):e46045. https://doi.org/ 10.1371/journal.pone.0046045

89. Conway JM, Walsh BH, Boylan GB, Murray DM (2018) Mild hypoxic ischaemic encephalopathy and long term neurodevelopmental outcome - a systematic review. Early Hum Dev 120:80-87. https://doi.org/10.1016/j.earlhumdev.2018.02. 007

90. Leidinger P, Backes C, Meder B, Meese E, Keller A (2014) The human miRNA repertoire of different blood compounds. BMC Genomics 15(1):474. https://doi.org/10.1186/1471-2164-15-474

91. Masè M, Grasso M, Avogaro L, D'Amato E, Tessarolo F, Graffigna A, Denti MA, Ravelli F (2017) Selection of reference genes is critical for miRNA expression analysis in human cardiac tissue. A focus on atrial fibrillation. Sci Rep 7:41127. https://doi org/10.1038/srep41127 https://www.nature.com/articles/ srep41127\#supplementary-information

92. Xiang M, Zeng Y, Yang R, Xu H, Chen Z, Zhong J, Xie H, Xu Y et al (2014) U6 is not a suitable endogenous control for the quantification of circulating microRNAs. Biochem Biophys Res Commun 454(1):210-214. https://doi.org/10.1016/j.bbrc.2014. 10.064

93. Pressler R, Auvin S (2013) Comparison of brain maturation among species: an example in translational research suggesting 
the possible use of bumetanide in newborn. Front Neurol 4:36-36. https://doi.org/10.3389/fneur.2013.00036

94. Roohey T, Raju TN, Moustogiannis AN (1997) Animal models for the study of perinatal hypoxic-ischemic encephalopathy: a critical analysis. Early Hum Dev 47(2):115-146

95. Murray DM, Boylan GB, Ryan CA, Connolly S (2009) Early EEG findings in hypoxic-ischemic encephalopathy predict outcomes at 2 years. Pediatrics 124(3):e459-e467. https://doi.org/ 10.1542/peds.2008-2190

96. Sarnat HB (1987) Hypoxic alterations in neonatal neurons: an acridine orange fluorochromic study of nucleic acids. Brain and Development 9(1):43-47

97. Looney A-M, Ahearne C, Boylan GB, Murray DM (2015) Glial fibrillary acidic protein is not an early marker of injury in perinatal asphyxia and hypoxic-ischemic encephalopathy. Front Neurol 6(264). https://doi.org/10.3389/fneur.2015.00264

98. Andrews S (2010) FastQC: a quality control tool for high throughput sequence data.

99. Team RC (2013) R: a language and environment for statistical computing.

100. Huber W, Carey VJ, Gentleman R, Anders S, Carlson M, Carvalho BS, Bravo HC, Davis S et al (2015) Orchestrating high-throughput genomic analysis with bioconductor. Nat Methods 12(2):115-121

101. Robinson MD, McCarthy DJ, Smyth GK (2010) edgeR: a bioconductor package for differential expression analysis of digital gene expression data. Bioinformatics 26(1):139-140

102. Law CW, Alhamdoosh M, Su S, Smyth GK, Ritchie ME (2016) RNA-seq analysis is easy as 1-2-3 with limma, Glimma and edgeR. F1000Research 5

103. Robinson MD, Oshlack A (2010) A scaling normalization method for differential expression analysis of RNA-seq data. Genome Biol 11(3):R25

104. Williams Z, Ben-Dov IZ, Elias R, Mihailovic A, Brown M, Rosenwaks Z, Tuschl T (2013) Comprehensive profiling of circulating microRNA via small RNA sequencing of cDNA libraries reveals biomarker potential and limitations. Proc Natl Acad Sci 110(11):4255-4260

105. Brennan GP, Vitsios DM, Casey S, Looney A-M, Hallberg B, Henshall DC, Boylan GB, Murray DM et al (2018) RNAsequencing analysis of umbilical cord plasma microRNAs from healthy newborns. PLoS One 13(12):e0207952. https://doi.org/ 10.1371/journal.pone.0207952

106. Chen Q, Xu J, Li L, Li H, Mao S, Zhang F, Zen K, Zhang CY et al (2014) MicroRNA-23a/b and microRNA-27a/b suppress Apaf-1 protein and alleviate hypoxia-induced neuronal apoptosis. Cell Death Dis 5:e1132. https://doi.org/10.1038/cddis.2014.92 https:// www.nature.com/articles/cddis201492\#supplementaryinformation

107. Kulshreshtha R, Davuluri RV, Calin GA, Ivan M (2008) A microRNA component of the hypoxic response. Cell Death Differ 15:667-671. https://doi.org/10.1038/sj.cdd.4402310. https://www.nature.com/articles/4402310\#supplementaryinformation

108. Ivan M, Harris Adrian L, Martelli F, Kulshreshtha R (2008) Hypoxia response and microRNAs: no longer two separate worlds. J Cell Mol Med 12(5a):1426-1431. https://doi.org/10. 1111/j.1582-4934.2008.00398.x

109. Ceolotto G, Giannella A, Albiero M, Kuppusamy M, Radu C, Simioni P, Garlaschelli K, Baragetti A et al (2017) miR-30c-5p regulates macrophage-mediated inflammation and proatherosclerosis pathways. Cardiovasc Res 113(13):1627-1638. https://doi.org/10.1093/cvr/cvx157

110. Oksuz Z, Serin MS, Kaplan E, Dogen A, Tezcan S, Aslan G, Emekdas G, Sezgin O et al (2015) Serum microRNAs; miR$30 c-5 p$, miR-223-3p, miR-302c-3p and miR-17-5p could be used as novel non-invasive biomarkers for $\mathrm{HCV}$-positive cirrhosis and hepatocellular carcinoma. Mol Biol Rep 42(3):713-720

111. Huang J, Yao X, Zhang J, Dong B, Chen Q, Xue W, Liu D, Huang Y (2013) Hypoxia-induced downregulation of miR-30c promotes epithelial-mesenchymal transition in human renal cell carcinoma. Cancer Sci 104(12):1609-1617

112. Zhu Y, Yu F, Jiao Y, Feng J, Tang W, Yao H, Gong C, Chen J et al (2011) Reduced miR-128 in breast tumor-initiating cells induces chemotherapeutic resistance via Bmi-1 and ABCC5. Clin Cancer Res 17:7105-7115

113. Papagiannakopoulos T, Friedmann-Morvinski D, Neveu P, Dugas J, Gill R, Huillard E, Liu C, Zong H et al (2012) Pro-neural miR128 is a glioma tumor suppressor that targets mitogenic kinases. Oncogene 31(15):1884-1895

114. Palumbo T, Faucz FR, Azevedo M, Xekouki P, Iliopoulos D, Stratakis CA (2013) Functional screen analysis reveals miR-26b and miR-128 as central regulators of pituitary somatomammotrophic tumor growth through activation of the PTEN-AKT pathway. Oncogene 32(13):1651-1659

115. Song B, Wang Y, Xi Y, Kudo K, Bruheim S, Botchkina GI, Gavin E, Wan Y et al (2009) Mechanism of chemoresistance mediated by miR-140 in human osteosarcoma and colon cancer cells. Oncogene 28:4065-4074. https://doi.org/10.1038/onc.2009.274. https://www.nature.com/articles/onc2009274\#supplementaryinformation

116. Tardif G, Hum D, Pelletier J-P, Duval N, Martel-Pelletier J (2009) Regulation of the IGFBP-5 and MMP-13 genes by the microRNAs miR-140 and miR-27a in human osteoarthritic chondrocytes. BMC Musculoskelet Disord 10(1):148. https://doi. org/10.1186/1471-2474-10-148

117. Li Q, Yao Y, Eades G, Liu Z, Zhang Y, Zhou Q (2013) Downregulation of miR-140 promotes cancer stem cell formation in basal-like early stage breast cancer. Oncogene 33:2589-2600. https://doi.org/10.1038/onc.2013.226. https://www.nature.com/ articles/onc2013226\#supplementary-information

118. Chen Y, Song Y, Wang Z, Yue Z, Xu H, Xing C, Liu Z (2010) Altered expression of MiR-148a and MiR-152 in gastrointestinal cancers and its clinical significance. J Gastrointest Surg 14(7): 1170-1179. https://doi.org/10.1007/s11605-010-1202-2

119. Muiños-Gimeno M, Espinosa-Parrilla Y, Guidi M, Kagerbauer B, Sipilä T, Maron E, Pettai K, Kananen L et al (2011) Human microRNAs miR-22, miR-138-2, miR-148a, and miR-488 are associated with panic disorder and regulate several anxiety candidate genes and related pathways. Biol Psychiatry 69(6):526-533. https://doi.org/10.1016/j.biopsych.2010.10.010

120. Taylor MA, Sossey-Alaoui K, Thompson CL, Danielpour D, Schiemann WP (2013) TGF- $\beta$ upregulates miR-181a expression to promote breast cancer metastasis. J Clin Invest 123(1):150 163. https://doi.org/10.1172/JCI64946

121. Visone R, Veronese A, Rassenti LZ, Balatti V, Pearl DK, Acunzo M, Volinia S, Taccioli C et al (2011) miR-181b is a biomarker of disease progression in chronic lymphocytic leukemia. Blood 118(11):3072-3079

122. Cai J, Guan H, Fang L, Yang Y, Zhu X, Yuan J, Wu J, Li M (2013) MicroRNA-374a activates Wnt/ $\beta$-catenin signaling to promote breast cancer metastasis. J Clin Invest 123(2)

123. Xu X, Wang W, Su N, Zhu X, Yao J, Gao W, Hu Z, Sun Y (2014) miR-374a promotes cell proliferation, migration and invasion by targeting SRCIN1 in gastric cancer. FEBS Lett 589(3):407-413. https://doi.org/10.1016/j.febslet.2014.12.027

124. Pescador N, Pérez-Barba M, Ibarra JM, Corbatón A, MartínezLarrad MT, Serrano-Ríos M (2013) Serum circulating microRNA profiling for identification of potential type 2 diabetes and obesity biomarkers. PLoS One 8(10):e77251. https://doi.org/ 10.1371/journal.pone.0077251 
125. Hegarty SV, Sullivan AM, O'Keefe GW (2016) Protocol for evaluation of neurotrophic strategies in Parkinson's disease-related dopaminergic and sympathetic neurons in vitro.

126. Shipley MM, Mangold CA, Szpara ML (2016) Differentiation of the SH-SY5Y human neuroblastoma cell line. J Vis Exp : JoVE 108:53193-53193. https://doi.org/10.3791/53193

127. Baars T, Skyschally A, Klein-Hitpass L, Cario E, Erbel R, Heusch G, Kleinbongard P (2014) microRNA expression and its potential role in cardioprotection by ischemic postconditioning in pigs. Pflugers Arch 466(10):1953-1961. https://doi.org/10.1007/ s00424-013-1429-3

128. Wang H, Wang J, Sun S, Wang Y, Guo J, Ning C, Yang K, Liu JF (2015) Identification of reference microRNAs for quantitative expression analysis in porcine peripheral blood mononuclear cells treated with polyinosinic-polycytidylic acid. Int J Immunogenet 42(3):217-225. https://doi.org/10.1111/iji.12198

129. Livak KJ, Schmittgen TD (2001) Analysis of relative gene expression data using real-time quantitative PCR and the 2(-Delta Delta C(T)) method. Methods (San Diego, Calif) 25(4):402-408. https:// doi.org/10.1006/meth.2001.1262

130. Vanhamme L, van den Boogaart A, Van Huffel S (1997) Improved method for accurate and efficient quantification of MRS data with use of prior knowledge. J Magn Reson 129(1): 35-43. https://doi.org/10.1006/jmre.1997.1244

131. Wong N, Wang X (2014) miRDB: an online resource for microRNA target prediction and functional annotations. Nucleic Acids Res 43(D1):D146-D152

132. Yuen T, Ruf F, Chu T, Sealfon SC (2009) Microtranscriptome regulation by gonadotropin-releasing hormone. Mol Cell
Endocrinol 302(1):12-17. https://doi.org/10.1016/j.mce.2008.12. 013

133. Dave RS, Khalili K (2010) Morphine treatment of human monocyte-derived macrophages induces differential miRNA and protein expression: impact on inflammation and oxidative stress in the central nervous system. J Cell Biochem 110(4):834-845. https://doi.org/10.1002/jcb.22592

134. Brors B, Gu L, Schlesner M, Eils R, Gu Z (2014) Circlize implements and enhances circular visualization in R. Bioinformatics 30(19):2811-2812. https://doi.org/10.1093/bioinformatics/btu393

135. Kuleshov MV, Jones MR, Rouillard AD, Fernandez NF, Duan Q, Wang Z, Koplev S, Jenkins SL et al (2016) Enrichr: a comprehensive gene set enrichment analysis web server 2016 update. Nucleic Acids Res 44(W1):W90-W97

136. Law CW, Chen Y, Shi W, Smyth GK (2014) Voom: Precision weights unlock linear model analysis tools for RNA-seq read counts. Genome Biol 15(2):R29

137. Ritchie ME, Phipson B, Wu D, Hu Y, Law CW, Shi W, Smyth GK (2015) Limma powers differential expression analyses for RNA-sequencing and microarray studies. Nucleic Acids Res 43(7):e47

138. Benjamini Y, Hochberg Y (1995) Controlling the false discovery rate: a practical and powerful approach to multiple testing. J Royal Stat Soc (Methodological):289-300

Publisher's Note Springer Nature remains neutral with regard to jurisdictional claims in published maps and institutional affiliations.

\section{Affiliations}

Sophie Casey ${ }^{1,2,3}$ (1) $\cdot$ Kate Goasdoue ${ }^{4} \cdot$ Stephanie M. Miller ${ }^{4} \cdot$ Gary P. Brennan ${ }^{5} \cdot$ Gary Cowin $^{6} \cdot$ Adam G. O'Mahony $^{3}$. Christopher Burke $^{7}$ - Boubou Hallberg ${ }^{8} \cdot$ Geraldine B. Boylan $^{1}$ • Aideen M. Sullivan ${ }^{3}$ - David C. Henshall ${ }^{5,9}$. Gerard W. O'Keeffe ${ }^{1,3} \cdot$ Catherine Mooney ${ }^{1,9,10} \cdot$ Tracey Bjorkman $^{4} \cdot$ Deirdre M. Murray ${ }^{1,2}$

1 Irish Centre for Fetal and Neonatal Translational Research (INFANT), University College Cork, Cork, Ireland

2 Department of Paediatrics and Child Health, University College Cork, Cork, Ireland

3 Department of Anatomy and Neuroscience, University College Cork, Room 2.33, Western Gateway Building, Cork, Ireland

4 Perinatal Research Centre, UQ Centre for Clinical Research, University of Queensland, Brisbane, Australia

5 Department of Physiology and Medical Physics, Royal College of Surgeons in Ireland, Dublin, Ireland
6 National Imaging Facility, Centre for Advanced Imaging, University of Queensland, Brisbane, Australia

7 Department of Pathology, Royal Brisbane and Women's Hospital, Brisbane, Australia

8 Neonatology, Karolinska University Hospital, Stockholm, Sweden

9 FutureNeuro Research Centre, Royal College of Surgeons in Ireland, Dublin, Ireland

10 School of Computer Science, University College Dublin, Dublin, Ireland 